\title{
Spin Foam Models and
}

\section{the Classical Action Principle}

\author{
Laurent Freidel $^{a}$, Kirill Krasnov ${ }^{b}$ \\ ${ }^{a b}$ Center for Gravitational Physics and Geometry \\ Department of Physics, \\ The Pennsylvania State University \\ University Park, \\ PA 16802, USA \\ freidel@phys.psu.edu \\ krasnov@phys.psu.edu \\ ${ }^{a}$ Laboratoire de Physique Théorique ENSLAPP \\ Ecole Normale Supérieure de Lyon \\ 46, allée d'Italie, \\ 69364 Lyon Cedex 07, France
}

\begin{abstract}
We propose a new systematic approach that allows one to derive the spin foam (state sum) model of a theory starting from the corresponding classical action functional. It can be applied to any theory whose action can be written as that of the BF theory plus a functional of the B field. Examples of such theories include BF theories with or without cosmological term, Yang-Mills theories and gravity in various spacetime dimensions. Our main idea is two-fold. First, we propose to take into account in the path integral certain distributional configurations of the B field in which it is concentrated along lower dimensional hypersurfaces in spacetime. Second, using the notion of generating functional we develop perturbation expansion techniques, with the role of the free theory played by the BF theory. We test our approach on various theories for which the corresponding spin foam (state sum) models are known. We find that it exactly reproduces the known models for BF and 2D Yang-Mills theories. For the BF theory with cosmological term in 3 and 4 dimensions we calculate the terms of the transition amplitude that are of the first order in the cosmological constant, and find an agreement with the corresponding first order terms of the known state sum models. We discuss implications of our results for existing quantum gravity models.
\end{abstract}

e-print archive: http://xxx.lanl.gov/abs/hep-th/9807092 


\section{Introduction}

Recently Baez [1], motivated by results [2] of the canonical (loop) approach to quantum gravity, introduced a notion of "spin foam models". The spin foam model of a theory, as we explain in details below, gives a way to calculate transition amplitudes for this theory. A transition amplitude arises as a sum over branched colored surfaces, or, using the name proposed by Baez, spin foams. The results of Reisenberger and Rovelli [2], Reisenberger [3], Barrett and Crane [4] indicate that quantum gravity might be constructed as a spin foam model.

However, spin foam models arise not only in the context of canonical (loop) quantum gravity. Indeed, many of the known TQFT's - for example, the Migdal-Witten model in two dimensions [5, 6], the Ponzano-Regge [7] and Turaev-Viro [8] models in three spacetime dimensions, the Ooguri model [9] and its generalizations by Crane, Kauffmann and Yetter [10] — are state sum models and, by considering instead of sums over colored triangulations sums over the corresponding colored dual complexes, can be formulated as spin foam models. The state sum models mentioned are believed to correspond to certain well-known topological field theories. However, a precise relation between the classical theory and the corresponding state sum model is not known for some of these models. Moreover, even for the models whose relation to the corresponding classical theory is established, this relation is rather indirect. Thus, summarizing, one can say that there exists a large class of spin foam models that are claimed to correspond to such classical theories as Yang-Mills, BF theories and gravity in various dimensions, but no precise relation to this effect is known in many cases.

The present paper is devoted to the study of this relation. More precisely, we study the relation between a spin foam formulation of a theory and the corresponding classical action principle. We propose and study a particular procedure that allows one to get a spin foam formulation for a large class of theories starting from the corresponcling action principle. Our approach is not limited to any particular spacetime dimension. However, having in mind applications to the models mentioned above, we restrict ourselves to two, three and four spacetime dimensions. ${ }^{1}$

When considering gravity, for simplicity, we restrict ourselves to metrics of Euclidean signature.

Our approach is inspired first of all by the results of the canonical (loop) approach $[12,13,14,15]$ to quantum gravity. Of great importance for us were also the ideas of Regge [16], the work of Baez [17] on degenerate solutions of general relativity and some results of Barrett [18]. Motivated by all these

\footnotetext{
${ }^{1}$ For an application of our procedure to higher-dimensional gravity see [11].
} 
results we propose to "approximate" some of the continuous fields appearing in the Lagrangian of the theory by distributional fields concentrated along lower dimensional hypersurfaces in spacetime. To make contact with the known lattice models, we put our distributional fields on a triangulated manifold, so that they are concentrated along polygons of the dual complex. However, the idea is also valid in a context more general than a lattice model. The action of the theory calculated on such distributional fields becomes a sum of certain integrals over the hypersurfaces of lower dimension. Then, to calculate a transition amplitude for the theory, one constructs the path integral of the exponentiated action over such distributional fields. As we shall see, this path integral becomes quite tractable.

To make this idea little more precise, let us specify the class of theories that can be treated within our approach. We consider theories whose action is BF-like, that is, whose action is of the form

$$
\int_{\mathcal{M}}[\operatorname{Tr}(E \wedge F)+\Phi(E)]
$$

where $E$ is the Lie algebra valued two-form field (for instance, $B$ field of BF theory), $F$ is the curvature of the connection form and $\Phi$ is certain (polynomial) function of the $E$ field, which can also depend on some Lagrange multipliers, as in the case of gravity in four dimensions, or on an additional background structure, as, for example, a fixed measure on $\mathcal{M}$ in the case of Yang-Mills theory in two dimensions. Below we will give many examples of theories belonging to this class. Thus, the action is that of the BF theory with an additional term. We call the $\mathrm{B}$ field $\mathrm{E}$ because of its relation with the non-abelian "electric" field of the canonical formulation.

In order to compute a transition amplitude for a theory belonging to this class, we take the path integral over classical configurations where the $E$ field, which is a zero-, one- or two-form in different dimensions, is a distributional field that vanishes everywhere except on two dimensional polygons in spacetime. Note that in our approach only these distributional configurations of the $\mathrm{E}$ field are taken into account in the path integral. No usual smooth configurations of the $\mathrm{E}$ field are summed over. This can be viewed as a discretization procedure where we "approximate" the smooth $E$ field by "squeezing" it into a distributional field on a collection of two-dimensional polygons. One can expect that this approximation becomes good as the twodimensional polygons become sufficiently packed in spacetime. However, this procedure can also be justified at a deeper level, as we shall argue.

First, a justification for considering distributional fields in the path integral comes from the canonical quantum theory. The phase space of all theories we work with, as it can be seen from the above action functional, 
is that of Yang-Mills theory. The space of quantum states of the canonical theory is then given by $L^{2}(\mathcal{A} / \mathcal{G})$, where $\mathcal{A} / \mathcal{G}$ is the space of connections on the spatial manifold modulo gauge transformations, and $L^{2}$ is defined by an appropriate choice of measure on this space. In the case the spatial manifold is one-dimensional, there exists quite a natural choice of this measure: the functionals on $\mathcal{A} / \mathcal{G}$ are just class functions, and it is natural to use the Haar measure on the group to define the inner product of such functionals. In higher dimensional cases, an analogous construction of $L^{2}(\mathcal{A} / \mathcal{G})$ was developed [14] within the approach of canonical (loop) quantum gravity. In this construction, integrable functions arise by considering the so-called cylindrical functionals, that is, functionals that depend on connection only through holonomies along some paths in space. In other words, one constructs the space of states of the theory by considering spaces of states of lattice gauge theories on graphs in space, and then taking the projective limit over graph refinements (see [14] for details). A characteristic feature of the quantum theory constructed with such a space of states is the distributional nature of the electric field operator. Let us illustrate this on the case of $(2+1)$ dimensional theory. A typical state from $L^{2}(\mathcal{A} / \mathcal{G})$ depends on the connection only through holonomies along some paths in space. Although this is not important for our heuristic consideration here, let us note that gauge invariance implies that the paths form a closed graph in space. Let us denote the edges of such a graph by $e_{i}$. Then a typical state supported on this graph is of the form

$$
\Psi\left(h_{e_{1}}(A), \ldots, h_{e_{n}}(A)\right),
$$

where $h_{e_{i}}(A)$ is the holonomy of $A$ along the edge $e_{i}$, and $\Psi$ is a function with sufficiently good behavior. The electric field $E$, which classically is a canonically conjugate quantity to $A$, quantum mechanically becomes the operator $\delta / \delta A(x)$ of variational derivative with respect to $A$. This operator, when acting on a typical state, gives zero for all points $x$ except the points lying on the edges $e_{i}$, where the result is distributional. Thus, in a typical quantum state, the electric field $E$ is distributional with support on edges $e_{i}$ of the corresponding graph. One normally views a state of the canonical theory as a state "at a given time". Thus, electric field "at each given time" is distributional and concentrated along edges of the graph. This suggests that one should think of the "history" of the electric field configuration as of a collection of two-dimensional surfaces in spacetime, along which the electric field is distributional. When sliced by a spatial hypersurface "at given time", such a collection of surfaces gives a graph in space, and electric field is distributional along the edges of this graph. These heuristic considerations suggest that one should consider configurations of $E$ field that are distributional and concentrated along two dimensional surfaces in spacetime. As we will see in more details below, the surfaces of support of $E$ field are always 


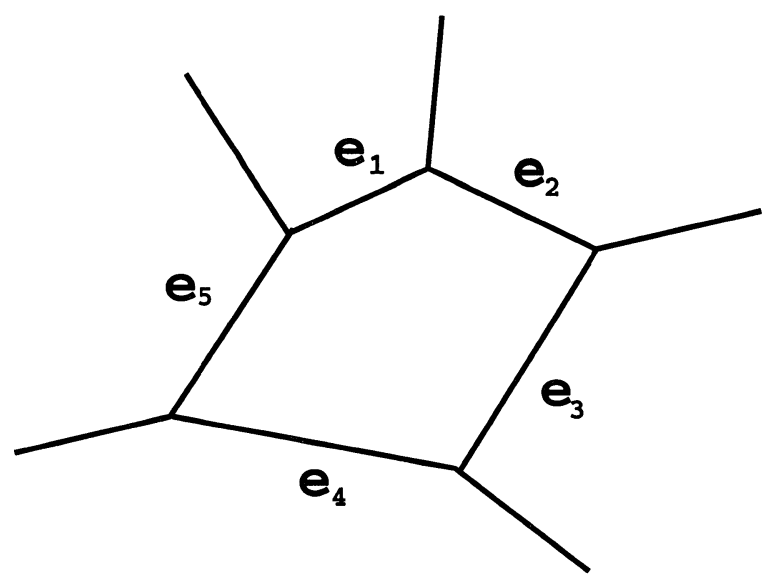

Figure 1: A typical quantum state of $(2+1)$ dimensional theory is labelled by a graph in space. The corresponding functional of connection depends on $A$ only through holonomies along edges $e_{i}$. The electric field in such state is distributional and concentrated along the edges $e_{i}$.

two-dimensional, independently of the dimension of the spacetime manifold.

The second justification for considering the path integral over such distributional $E$ fields comes from the fact of existence of similar distributional solutions of the equations of motion in the classical theory. As it was shown by Baez [17] for the case of gravity, there exist solutions of the classical equations of motion in which the $E$ field is distributional and concentrated along two-dimensional surfaces in spacetime. In these solutions the $E$ field is zero except in a tubular neighborhood of a two-dimensional surface in spacetime. The connection field away from the surface then must be either arbitrary or flat, depending on the detailed form of the Lagrangian of the theory. Solutions of this type can be shown to exist for a large class of theories. Here we take the fact of the existence of such solutions as a motivation for our construction. Namely, we know that the simplest way to approach the computation of a path integral is to consider the semi-classical approximation. Then the Feynman path integral is evaluated as an integral over the classical moduli space (space of classical solution) with a measure given by the one loop expansion of the action around the classical solution. In this case, there may be different phases in the theory, and the type of solutions that dominate the path integral may be different in different phases. Thus, there may exist a phase in a theory of the type we study where the path integral is dominated by the distributional solutions found by Baez. At this point it is instructive to make an analogy with the usual Yang-Mills theories. In (abelian) Yang-Mills theories one also finds "distributional" solutions of 
the classical equations of motion: monopole solutions. In Dirac's way [19] of treating the monopole, it is described by a configuration of fields in which magnetic field is distributional along certain lines. Distributional monopole solutions are not in the spectrum of perturbative quantum theory. However, one must include them when calculating the partition function of the theory via path integral method to reproduce correctly properties of certain phases of the theory, such as the confining phase. In fact, the confining phase of the theory is the one in which monopole solutions dominate the path integral [20]. Distributional solutions of the type discovered by Baez [17] are very similar in nature to monopole solutions. In fact, they are close to dual monopoles: electric field is distributional and concentrated along flux lines in space, instead of the magnetic field. Thus, the analogy with monopole solutions suggests that one should include these distributional solutions when taking the path integral. In this paper we include only these distributional solutions, neglecting the usual smooth solutions playing the role in perturbative quantum theory. Thus, we attempt to study the phase of various theories in which the distributional solutions dominate the path integral. This is the motivation for considering the path integral over distributional configurations of $E$ field.

The last but not least justification of our approach is the test of the procedure on the exact results coming from TQFT's. As we shall see, for a large class of theories our approach gives results that are in a good agreement with the known state sum models.

In this paper we only calculate path integrals over the distributional fields "living" on a fixed collection of surfaces in spacetime. In the case of topological theories, the result for a particular fixed collection of surfaces does not depend on the collection. One cannot expect this property to hold for such theories as gravity, which is not a topological theory. Thus, one might want to perform a sum over all possible collections of surfaces in spacetime, or take a limit as the triangulation becomes more refined. We do not address this, very important, problem in the present paper.

Usual interactive quantum field theories have been successfully understood using perturbative expansion in terms of Feynman graphs. In this case the interacting QFT is considered as a perturbation of a free field theory. From a technical point of view, what makes this approach successful is the exact solvability of the free field theory, i.e., our ability to compute all possible correlation functions. This ability can be recasted into the knowledge of the generating functional, and Feynman graphs can be viewed as the evaluation of certain differential operators acting on the generating functional. 
We will employ a somewhat similar strategy. In order to calculate transition amplitudes for our class of theories we will use the machinery of generating functionals. In our approach the role of the "free" field theory is played by the BF theory and the terms in the action polynomial in $E$ are analogous to the "interaction" terms. The idea to use the BF theory as a "free" field theory is not new: it has been applied with some success in the context of Yang-mills theories (see [21]). However, the details of how the BF theory is used in our approach differ from those of [21]. In order to calculate a transition amplitude, i.e., the path integral of the exponentiated action

$$
\int \mathcal{D} A \mathcal{D} E e^{i \int[\operatorname{Tr}(E \wedge F)+\Phi(E)]},
$$

we formally rewrite this path integral as

$$
\left(e^{i \int \Phi\left(\frac{\delta}{i \delta J}\right)} Z[J]\right)_{J=0},
$$

where

$$
Z[J]:=\int \mathcal{D} A \mathcal{D} E e^{i \int[\operatorname{Tr}(E \wedge F)+\operatorname{Tr}(E \wedge J)]} .
$$

We shall refer to $Z[J]$ as the generating functional. Here $J$ is a Lie algebra valued two form field. Using terminology from field theory we will call the $J$ field current. One of our main results is the exact computation, in the context of spin foam models, of the generating functional $Z$. It is obtained by integrating over fields $A, E$ that "live" on a fixed triangulation of the spacetime manifold. Then the transition amplitude for any theory of the type we consider is given as a formal power series in variational derivatives $\delta / \delta J$ with respect to the current field. The series we get are quite reminiscent of the usual Feynman diagram expansions. Thus, the powerful technique of generating functionals allows us to study different theories - such as BF theory and gravity - from the same point of view. We note that, to the best of our knowledge, the technique of generating functionals has never before been used in the context of spin foam models.

In cases when the spacetime manifold is two, three and four-dimensional, for each fixed triangulation we find an explicit expression for the generating functional. We then use the obtained expressions to calculate the transition amplitudes for various theories. We find that our approach exactly reproduces the state sum models of $2 \mathrm{D}$ Yang-Mills and BF theory in any dimension. For BF theories with cosmological term we compare the amplitude obtained within our approach with the one given by the corresponding state sum model by decomposing them into the power series in the cosmological constant and comparing the first order terms. Again, we find an agreement. We make some comments as to consequences of our results for the quantum gravity models in four spacetime dimensions. 
The paper consists of two main parts. In the first part, containing this section and Sec.2, we introduce the path integral techniques in the context of spin foam models. Here we also introduce the generating functional, and compute it in various spacetime dimensions. The second part contains applications of the general techniques developed earlier to concrete theories. This also serves as a test of our approach, for one can compare the spin foam models obtained by our techniques with the known state sum models. In Sec. 3 we define the class of theories that can be treated by techniques described in this paper, and give examples of physically interesting theories belonging to this class. Sec.4 describes briefly some known state sum models and summarizes the known arguments that relate the state sum models to the classical theories. Here we also obtain some properties of various state sum models that will be needed later. Finally, using the generating functional technique, we derive in Sec.5 the spin foam models corresponding to various theories of Sec. 3 and compare them with the known models. We conclude with a discussion.

\section{Generating functional}

As we briefly explained in the Introduction, to calculate transition amplitudes for a theory of the type we consider here it is very convenient to first calculate the generating functional of BF theory. Then transition amplitudes can be obtained as formal power series in derivatives with respect to the current. In this section we calculate the generating functional of the $\mathrm{BF}$ theory, i.e., the path integral

$$
Z[J]=\int \mathcal{D} A \mathcal{D} E e^{i \int[\operatorname{Tr}(E \wedge F)+\operatorname{Tr}(E \wedge J)]}
$$

in two, three and four spacetime dimensions. Here $A$ is a connection on some $G$ bundle over the spacetime manifold $\mathcal{M}$, where $G$ is the gauge group of the theory, $F$ is the curvature 2-form of the connection, and $E, J$ are Lie algebra valued (d-2)-form fields, where $\mathrm{d}$ is the dimension of $\mathcal{M}$. For simplicity, we consider here only the case when the spacetime manifold $\mathcal{M}$ is closed. We also restrict ourself to the case of the gauge group being $G=\mathrm{SU}(2)$. Although for practical applications of spin foam models one is usually interested in the case when $\mathcal{M}$ has a boundary consisting of "initial" and "final" spatial hypersurfaces, we do not consider this here because our main goal is to compare the spin foam models obtained by our techniques with the known state sum models, which can be done already in the case of closed $\mathcal{M}$. At any rate, the inclusion of boundaries is straightforward, and all our formulas can be easily generalized to this case. To give meaning to the 
path integration over spacetime fields $A, E$, we replace the $E$ field by certain distributional field concentrated over two-dimensional surfaces in spacetime. To make contact with the known state sum models we put our fields on the 2-dimensional cellular complex dual to some triangulation of the spacetime manifold. In each particular calculation this triangulation is fixed and all results explicitly depend on it. We first investigate the generating functional in a general case, without specifying the dimension of spacetime manifold, and then find an explicit expression for it in each particular dimension.

\subsection{General framework}

Let us fix a triangulation $\Delta$ of a d-dimensional compact oriented spacetime manifold $\mathcal{M}$. This triangulation defines another decomposition of $\mathcal{M}$ into cells called dual complex. There is one-to-one correspondence between $k$ simplices of the triangulation and $(\mathrm{d}-\mathrm{k})$-cells in the dual complex. We orient each cell of the dual complex in an arbitrary fashion, which also defines an orientation for all simplices of the triangulation. The (d-2)-form $E$ can now be integrated over the (d-2)-simplices of the triangulation the result being a collection of the Lie algebra elements $X$ - one vector $X \in \mathrm{su}(2)$ for each 2cell dual to a (d-2)-simplex of the triangulation. We would like to discretize our model by replacing the continuous $E$ field by the collection of the Lie algebra elements $X$. It turns out, however, that this is not yet the most convenient set of variables for the theory. For reasons which we give below, we use another, more convenient set of variables introduced by Reisenberger $[22]$.

In what follows we will often call 2-cells of the dual triangulation dual faces. Instead of a single $X$ for each dual face, we introduce a set of variables, which we call $X_{w}$. To do this, we divide each dual face into what Reisenberger calls "wedges" (see Fig. 2). To construct wedges of the dual face one first has to find the "center" of the dual face. This is the point on the dual face where it intersects with the corresponding (d-2)-simplex of the triangulation. One then has to draw lines connecting this center with the centers of the neighboring dual faces. The part of the dual face that lies between two such lines is exactly the wedge. Thus, each dual face splits into wedges, and we assign a Lie algebra element $X_{w}$ to each wedge $w$. All variables $X_{w}$ that correspond to a single dual face are required to have the same length. Recall that vectors of the same length only differ by a gauge transformation. Wedges of a given dual face are in one-to-one correspondence with the d-simplices of the triangulation neighboring this dual face. Thus, the physical meaning of each variable $X_{w}$ can be said to be the integral of $E$ over the (d-2) simplex of the triangulation "from the point of view" of a 


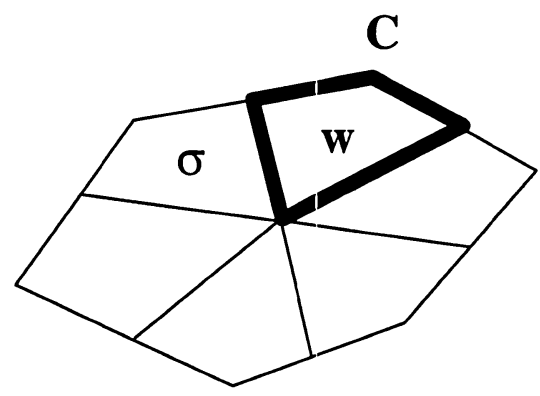

Figure 2: A face $\sigma$ of the dual triangulation. The portion of $\sigma$ indicated by bold lines is what is called wedge here. The point labelled by $C$ is the center of one of the d-simplices neighbored by $\sigma$.

particular d-simplex containing this (d-2)-simplex. In other words, with the introduction of the wedge variables $X_{w}$ we allow integrals of $E$ over one and the same (d-2)-simplex of triangulation to be different when this simplex is considered as belonging to different d-simplices, as long as lengths of all such $X_{w}$ coincide. Note that the number of variables $X_{w}$ that arises this way for a given triangulation is equal to the number of $d$-simplices times the number of (d-2)-simplices in each d-simplex.

Having discussed the geometrical meaning of the wedge variables $X_{w}$ we are ready to introduce the distributional $E$ fields. Heuristically, our procedure of replacement of a smooth $E$ field by a distribution concentrated along the wedges amounts to "squeezing" of the smooth $E$ field that is "spread" over a (d-2) simplex of the triangulation to a single point on this (d-2) simplex, the point where the simplex intersects with the 2-cell of the dual complex. Thus, we define a distributional field $E_{w}$ concentrated along a wedge $w$ to be a 2 -form satisfying the following relation:

$$
\int_{\mathcal{M}} \operatorname{Tr}\left(E_{w} \wedge J\right)=\operatorname{Tr}\left(X_{w} \int_{w} J\right)
$$

Here $J$ is any $\operatorname{Ad}(P)$-valued 2-form, and $w$ stands for a wedge. The integral on the right-hand-side is performed over the wedge $w$. This, in particular, implies that the Lie algebra element $X_{w}$ is equal to the integral of $E_{w}$ over the (d-2)-simplex of the triangulation that is dual to the 2-cell $\sigma$. We then define distributional field $E$ to be

$$
E=\sum_{w} E_{w}
$$

Although with the introduction of the wedge variables we have increased the number of independent variables in the theory, one can still argue that 
the physical content of the theory should be the same because the norm of all the $X_{w}$ belonging to one and the same dual face are required to be equal. For the case of BF theory one can directly prove that the introduction of the independent wedge variables does not change the theory in the case the topology of dual faces $\sigma$ is that of a disc, which is what we will assume here. For more complicated theories with non-zero "interaction" term (the term in the action that makes the theory to be different from the BF theory), the wedge variables are so indispensable that it is not clear whether one can even define the theory without these variables. It is interesting, however, that, even in the case of BF theory one has to use the wedge variables if one wants to reproduce a triangulation-independent state sum model for the case the topology of $\sigma$ is different from that of a disc. This serves as a strong motivation for considering the wedge variables. Another motivation for using them is, as we explain below, that the wedge variables appear naturally when one tries to implement the gauge symmetry in the theory. Also, as we shall see, using these variables we will be able to reproduce the well-known state sum models in various dimensions. Thus, we are going to use the set of wedge variables motivated, in particular, by the results we obtain.

The discretization we consider is consistent with the results of the canonical approach. The restriction of $E$ constructed as described above to a generic "spatial" ((d-1)-dimensional) slice $\Sigma$ of $\mathcal{M}$ is non-zero only along edges of the graph $\Gamma$ in $\Sigma$, where $\Gamma$ is the intersection of the dual complex with $\Sigma$. Thus, this "discretization" realizes rather explicitly Faraday's idea of lines of electric force. Indeed, the restriction of $E$ on a spatial hypersurface can be described as a collection of flux lines of non-abelian electric field. It is not hard to see what states would correspond to such $E$ in the quantum theory. Recall that the quantum states are functionals $\Psi[\underline{A}]$, where $\underline{A}$ is the pullback of the connection field $A$ on the spatial hypersurface $\Sigma$. The densitized vector field $\tilde{E}$ (that is dual to $\underline{E}$ ) becomes the operator of variational derivative with respect to $\underline{A}$. In order for $\tilde{E}$ in a state $\Psi[\underline{A}]$ to be zero everywhere except along the edges of $\Gamma$ the state $\Psi$ should depend on the values of the connection $\underline{A}$ only along the edges. Note also that our construction of the distributional $E$ field gives us $\tilde{E}$ whose length is constant along each edge. This means that the state $\Psi$ can depend on the values of $\underline{A}$ only as a function of the holonomies of the connection along the edges. These are exactly the states one finds in the canonical approach to quantum heory, as we explained in the introduction.

To calculate the generating functional $Z$ as a function of a fixed triangulation $\Delta$, we have to take the integral of the exponentiated action over the "discretized" dynamical fields $A, E$, that is, the fields "living" on the 


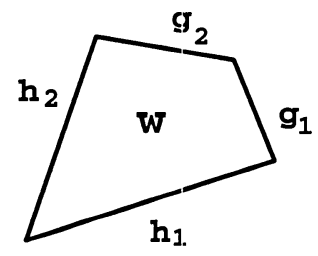

Figure 3: A wedge $w$ of the dual face and the group elements: holonomies along the edges of $w$.

triangulation $\Delta$. The "discretized" action, that is, the action evaluated on the distributional field $E$, becomes a function of the Lie algebra elements $X$, and a functional of connection $A$ and current $J$. Using (2),(3), we find that this discretized action is given by

$$
\sum_{w} \int_{w}\left[\operatorname{Tr}\left(F X_{w}\right)+\operatorname{Tr}\left(J X_{w}\right)\right]
$$

where the sum is taken over the wedges of faces of the dual complex (' $w$ ' stands here for a wedge), and the integral is performed over each wedge, the integrand being the curvature of the connection $A$ contracted with the Lie algebra element $X_{w}$ "living" on that wedge plus the current $J$ contracted with $X_{w}$. Each integral is performed using the orientation of the dual face to which the wedge belongs.

We could now substitute this discretized action into the path integral and integrate over $X$ and $A$. However, we first have to discuss what measure has to be used to integrate over $X, A$. To give meaning to the integration over $A$, let us replace a continuous field $A$ by a collection of group elements. To do this we use the following approximation

$$
\int_{w} \operatorname{Tr}\left(F X_{w}\right) \approx \operatorname{Tr}\left(Z_{w} X_{w}\right)
$$

where $Z_{w}$ is the Lie algebra element corresponding to the holonomy of $A$ around the wedge (see Fig. 3). The base point of the holonomy is not fixed at this stage (see below). In other words,

$$
\exp Z_{w}=g_{1} h_{1} h_{2} g_{2},
$$

where $g_{1}, h_{1}, h_{2}, g_{2}$ are the holonomies of $A$ along the four edges that form the boundary of the wedge $w$. We assume a local trivialization of the bundle over $w$ so that the holonomies are group elements. The order in which the product of group elements is taken is determined by the orientation of the 
dual face. Of course, $\exp Z_{w}$ is defined only up to its conjugacy class (any of the four edges can be taken to be the "first"). Thus, there is an ambiguity in the choice of the base point for the holonomy, which we have to fix in some way. To fix it we define a notion of "discretized" gauge transformation and fix the ambiguity requiring the discrete action to be gauge invariant.

But first, let us replace the continuous current field $J$ by a collection of Lie algebra elements $J_{w}$ :

$$
J_{w}:=\int_{w} J .
$$

Here, to perform the integration, a trivialization of the bundle over the wedge $w$ is chosen. The discretized action now becomes

$$
\sum_{w} \operatorname{Tr}\left(Z_{w} X_{w}\right)+\operatorname{Tr}\left(J_{w} X_{w}\right) .
$$

We fix a definition of $Z_{w}$ in such a way that this action is gauge invariant.

We define the discrete gauge transformation so that it "acts" in the center of each d-simplex. More precisely, a gauge transformation is parameterized by a collection of group elements: a group element $g$ for each d-simplex. First of all, the gauge transformation is defined for the holonomy $U$ of the connection along any loop that starts and finishes at the center $C$ of the $\mathrm{d}$-simplex. The transformation is as follows:

$$
U \rightarrow g U g^{-1} .
$$

The wedge variables $X_{w}$ and the discrete current variables $J_{w}$ transform as

$$
X_{w} \rightarrow g X_{w} g^{-1}, \quad J_{w} \rightarrow g J_{w} g^{-1} .
$$

With this definition $\operatorname{Tr}\left(X_{w} Z_{w}\right)$ is gauge invariant only when $\exp Z$ is defined as the holonomy around the wedge $w$ whose starting and final point is the center $C$ of the d-simplex, as in (6). This fixes the ambiguity in $Z_{w}$. With $Z_{w}$ defined this way the discretized action is gauge invariant.

The approximation (5) is good for $Z_{w}$ close to zero element in the Lie algebra. Thus, this approximation is certainly justified for BF theory, where we expect only connections close to flat to matter in the quantum theory. It is harder to justify this approximation for theories, for which the classical equations of motion do not imply the connection to be flat, as is the case, for example, for BF theory with cosmological term or for gravity. However, even for such theories, one would expect the approximation (5) become better as the triangulation of the manifold becomes finer and the dual faces become smaller. In quantum gravity we expect finer triangulations to matter most, 
which can serve as a justification for the above approximation in the case of gravity. The approximation (5) will be additionally justified when we discuss the problem of the integration over $X_{w}$ variables. To define the later we will refer to a certain standard field theory calculation for BF theory in two dimensions. As we shall see, the approximation (5) is quite natural from the point of view of $2 \mathrm{D} \mathrm{BF}$ theory.

The approximation (5) finishes the discretization procedure for the classical action. We can now calculate the path integral for the generating functional $Z$ by integrating the exponentiated discrete action (8) over the Lie algebra elements $X_{w}$ and group elements $g, h$. This path integral is given by

$$
Z(J, \Delta)=\prod \int_{\mathrm{SU}(2)} d g \prod \int_{\mathrm{SU}(2)} d h \int \prod_{w} d X_{w} e^{i \sum_{w} \operatorname{Tr}\left(X_{w} Z_{w}\right)+\operatorname{Tr}\left(X_{w} J_{w}\right)} .
$$

Here the integrals are taken over all group elements $g, h$, entering the expression through $Z_{w}$, see (5). These integrals form the discrete analog of $\mathcal{D} A$, $d g$ is the normalized Haar measure $\int d g=1$ on $\mathrm{SU}(2)$. The integrals over $X_{w}$ present here - one for each edge - form the analog of the integral over $\mathcal{D} E$. The measure $d X$ here is some measure on the Lie algebra. For now, we will leave this measure unspecified. Recall now that the integrals over $X_{w}$ are not independent: all $X_{w}$ that belong to one and the same dual face should have the same norm. However, as we shall see, there is no need to impose these constraints: one can integrate over all $X_{w}$ independently, but later, when one integrates over the group elements, only the contributions that come from $X_{w}$ of an equal norm will survive. Thus, we do not impose these constraints in (11).

Let us now investigate the structure of the path integral (11). To calculate $Z$ we have to find the function of $\exp Z_{w}, \exp J_{w}$ that is given by

$$
\int d X_{w} e^{i \operatorname{Tr}\left(X_{w} Z_{w}+X_{w} J_{w}\right)}
$$

In fact, it is not hard to see that this function is proportional to the $\delta$-function of $\exp Z_{w}$ peaked at $\exp J_{w}$. The proportionality coefficient can be a gauge invariant function of $J_{w}$. As we explain below, this function must be set to be equal to $P\left(J_{w}\right)$, where $P$ is the function that relates Lebesgue measure on the Lie algebra and Haar measure on the group (see the Appendix B). Thus, as the result of (12) we get:

$$
P\left(J_{w}\right) \delta\left(\exp Z_{w} \exp J_{w}\right),
$$

where $\delta$ is the standard $\delta$-function on the group. Thus, the function of 
$Z_{w}, J_{w}$ given by (12) can be written as

$$
P\left(J_{w}\right) \sum_{j} \operatorname{dim}_{j} \chi_{j}\left(\exp Z_{w} \exp J_{w}\right),
$$

where we used the well-known decomposition of $\delta$-function on the group into the sum over characters $\chi_{j}\left(\exp Z_{w} \exp J_{w}\right)$ of irreducible representation. Here the sum is taken over all irreducible representations of SU(2) (labelled by spins $j)$, and $\operatorname{dim}_{j}=(2 j+1)$.

Thus, we find that

$$
Z(J, \Delta)=\prod \int_{\mathrm{SU}(2)} d g \prod \int_{\mathrm{SU}(2)} d h \prod_{w} P\left(J_{w}\right) \sum_{j_{w}} \operatorname{dim}_{j_{w}} \chi_{j_{w}}\left(\exp Z_{w} \exp J_{w}\right) .
$$

The integration over the group elements can now be easily performed using the well-known formulas for the integrals of the products of matrix elements. However, let us first give a systematic derivation of the result (13).

To find the result of the integral (12) we relate it to a more complicated integral, for which a precise result is known from 2D BF theory calculation. Recall that so far the measure $d X_{w}$ in (12) is unspecified. The following relation to $2 \mathrm{D} \mathrm{BF}$ theory will also serve the purpose of specifying this measure. Let us restrict our attention to a particular wedge $w$. We can restrict the bundle $\mathcal{P}$ to $w$ and get an $\mathrm{SU}(2)$ bundle $\mathcal{P}_{w}$ over $w$. Let $A$ be a connection on $\mathcal{P}_{w}$, and $E$ be an $\operatorname{Ad}(\mathcal{P})$ valued 0 -form. Consider the following path integral:

$$
\int \mathcal{D} A \mathcal{D} E \exp \left(i \int_{w}[\operatorname{Tr}(F E)+\operatorname{Tr}(J E)]\right)
$$

where the integration over $A$ is performed subject to the condition that the connection on the boundary of $w$ is fixed, and $J$ is given by

$$
J=\delta(p) J_{w}
$$

where $p$ is an arbitrary fixed point on $w$, and $J_{w}$ is the same as in (12),(13). The path integral (16) is just a partition function of BF theory on the disk with the distributional source given by (17). This partition function can be derived using results of [23]. The result is given by (13), where $Z_{w}$ is the Lie algebra element that corresponds to the holonomy of $A$ along the boundary of $w$. We will not present this calculation here. Instead, we refer to the calculation performed in [23] for the partition function of 2D BF theory on a punctured sphere. The result (13) can then be checked by taking the partition function on the disk (equal to $\delta(g)$, where $g$ is the holonomy along the boundary of the disk), and integrating it with (13) over $d g$. This must give the partition function on a punctured sphere, and indeed reproduces the 
result given in [23]. The only cautionary remark we have to make is that the calculation performed in [23] finds a gauge invariant partition function, that is, the one in which one takes $J=\delta(p) h J_{w} h^{-1}$ and integrates over $d h$. The techniques developed in [23] can be used only to calculate gauge invariant quantities, and are not directly applicable to the integral (16). Thus, strictly speaking, using the results of [23] one can only argue that (16) is equal to

$$
P\left(J_{w}\right) \delta\left(e^{Z_{w}} h e^{J_{w}} h^{-1}\right),
$$

where $h$ is some group element. To get rid of $h$ in this expression, and, thus, to get (13), we will recall how "discretized" gauge transformations act on the Lie algebra elements $Z_{w}, J_{w}$, see (9),(10). The result of (12) must be invariant under this gauge transformations. It is not hard to see that this fixes $h$ above to be unity, thus, giving (13).

Having discussed how one can calculate the path integral (16), let us now show that this path integral is, in fact, equivalent to (12). Indeed, the integration over $E$ in (16) can be performed in two steps. First, one integrated over $E(x), x \neq p$, then one integrates over $E(p)$ :

$$
\int d X_{w} \int_{E(p)=X_{w}} \mathcal{D} E \int \mathcal{D} A \exp \left(i \int_{w}[\operatorname{Tr}(F E)+\operatorname{Tr}(J E)]\right) .
$$

Since the current is distributional and concentrated at point $p$, the last term in the exponential does not matter when one integrates over $\mathcal{D} E, \mathcal{D} A$. On the other hand, using the same approximation as the one used in (5), it is not hard to show that

$$
\int_{E(p)=X_{w}} \mathcal{D} E \int \mathcal{D} A \exp \left(i \int_{w} \operatorname{Tr}(F E)\right),
$$

where the integral over $A$ is taken with $A$ on the boundary of $w$ fixed, is approximated by

$$
e^{i \operatorname{Tr}\left(Z_{w} X_{w}\right)},
$$

where $\exp Z_{w}$ is the holonomy of $A$ along the boundary of $w$. Putting this back to (19) one gets exactly (12). This finishes our discussion of the derivation of (13).

Having discussed the derivation of the expression (15) for the generating functional, we can now perform the integrals over the group elements. Integration over the group elements $h$ that correspond to edges dividing dual faces into wedges is the same in any dimension, and we perform it here. The rest of the group elements corresponds to edges that form the boundary of the dual faces. The integration over these group elements $g$ is different in different dimensions, and we perform it in the following subsections. Each 


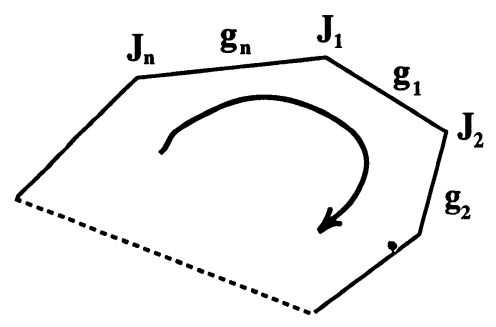

Figure 4: The product of the group elements is the holonomy around the dual face $\sigma$ with the insertions of the group elements $\exp J$ at each center of the corresponding $d$-simplex.

group element $h$ is "shared" by two wedges; thus, we have to take the integral of the product of two matrix elements. Such an integral is given by (142) of the Appendix. Integrating over all these edges, and making a simple change of variables to eliminate trivial integrations, we find

$$
\begin{array}{r}
Z(J, \Delta)=\prod_{\epsilon} \int_{\mathrm{SU}(2)} d g_{\epsilon} \times \\
\prod_{\sigma} \sum_{j_{\sigma}}\left(\operatorname{dim}_{j_{\sigma}}\right)^{\kappa_{\sigma}} P\left(J_{1}\right) \cdots P\left(J_{n}\right) \chi_{j_{\sigma}}\left(\exp J_{1} g_{\epsilon_{1}} \exp J_{2} \cdots \exp J_{n} g_{\epsilon_{n}}\right) .
\end{array}
$$

Here the remaining integrals are over the group elements $g_{\epsilon}$ that correspond to the edges $\epsilon$ of the dual complex (edges that connect centers of 4 -simplices). The second product is taken over the dual faces $\sigma ; j_{\sigma}$ is the spin labelling the dual face $\sigma, \kappa_{\sigma}$ is the Euler characteristics of $\sigma$. It is equal to unity in case the dual face has the topology of a disc. In what follows we will always assume that this is the case. The order of the group elements in the argument of $\chi_{j_{\sigma}}$ is clear from the Figure 4.

Note that in the integration we just performed survived only the terms for which the spins of all wedges corresponding to one and the same dual face are equal. In (22) this spin, which is the same for all wedges belonging to the same dual face, is denoted by $j_{\sigma}$. As one can see from the formula (137) for the inverse Fourier transform, the spin labelling a wedge in (15) has the meaning of the length of the corresponding Lie algebra element $X$. Thus, as we said above, there is no need to impose the constraint that length of all wedge variables $X_{w}$ belonging to one and the same dual face are equal: this constraint gets imposed automatically when the integration over the group elements is performed.

The expression (22) for the generating functional can be further simplified by integrating over the group elements $g_{\epsilon}$. However, the result of 
this integration is different in different dimensions, and we will perform it separately for each particular case.

Before we calculate the generating functional for each particular spacetime dimension, let us pause to discuss some general properties of the expression for $Z(J, \Delta)$. The generating functional has several symmetries which we describe as follows. First, the generating functional is invariant under gauge transformations. Namely, let us consider a transformation $J_{w} \rightarrow g_{h(w)} J_{w} g_{h(w)}^{-1}$, where $h(w)$ denotes the d-simplex of $\Delta$ to which $w$ belongs. Here $g_{h(w)}$ is the same for all $w$ belonging to the simplex $h$. The generating functional (11) satisfies:

$$
Z\left(g J g^{-1}, \Delta\right)=Z(J, \Delta) .
$$

This "discrete" gauge transformation is parameterized by one group element for each d-simplex of $\Delta$. However the expression (22) for the generating functional has a bigger invariance. Namely, let us denote by $e_{+}(w), e_{-}(w)$ the two edges of the wedge $w$ which meet at the center of the d-simplex $h(w)$. Let us associate with each edge $e_{ \pm}(w)$ a group element $g_{e_{ \pm}(w)}$ so that it is one and the same for different wedges $w$ when $e_{ \pm}(w)$ is one and the same. The transformation of all currents according to $J_{w} \rightarrow g_{e_{+}(w)} J_{w} g_{e_{-}(w)}^{-1}$ leaves the generating functional invariant. This transformation, which is parameterized by $d+1$ group elements per simplex, contains the gauge transformation as its particular case. The later corresponds to $g_{e_{+}(w)}=g_{e_{-}(w)}=g_{h(w)}$ for all $w$ that belong to $h$. The appearance and significance of the described extra symmetry is not yet clear to us.

The generating functional is covariant under the diffeomorphism group. Let $f$ denote a diffeomorphism of the underlying manifold $\mathcal{M}$, and denote by $f(\Delta)$ the image of the embedded triangulation $\Delta$ under the diffeomorphism. If $J_{w}=\int_{w} J$, let us denote $f^{*} J \equiv \int_{f(w)} J=\int_{w} f^{*} J$. Then

$$
Z(J, f(\Delta))=Z\left(f^{*} J, \Delta\right) \text {. }
$$

The generating functional is generally not invariant under a refinement of the triangulation $\Delta$ unless the theory is a topological field theory (unless $J=0)$.

To define the generating functional we had to choose an arbitrary orientation of the wedges. However, the generating functional is independent of the orientation chosen. If one, for example, reverses the orientation of the wedge $w$ this results: (i) in the change $J_{w}=\int_{w} J$ into $-J_{w}$; (ii) it changes the holonomy of the connection along the wedge into its inverse. The two effects cancel each other leaving the generating functional invariant. 


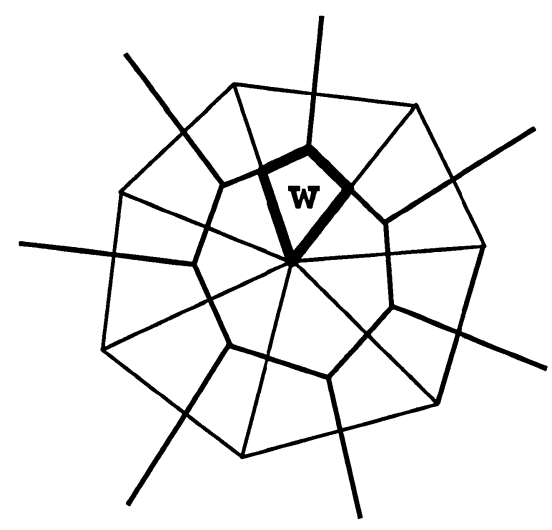

Figure 5: Part of a triangulation of 2-dimensional spacetime manifold, with one face of the dual complex shown. Bold lines indicate a wedge of the dual face.

\subsection{2 dimensions}

The case of two spacetime dimensions is somewhat special in the sense that dual faces (2-cells) cover the manifold (see Fig. 5). The general construction of the previous subsection prescribes to replace the continuous field $E$, which in the case of two dimensions is a zero form, by a distributional field concentrated along dual faces. However, since the spacetime is two-dimensional, the dual faces cover the manifold, and we just have to replace the $E$ field by a field constant on each wedge, and equal to the Lie algebra element $X_{w}$.

Let us now see that it is quite a reasonable thing to do from the point of view of the canonical quantum theory. In the case $\mathcal{M}$ is compact, which is the case of interest for us here, a spatial slice $\Sigma$ of $\mathcal{M}$ consists of a finite number of circles $S^{1}$. The states of the canonical theory are just class functions of the holonomies of $\underline{A}$, which is the pullback of $A$ on $\Sigma$, around this circles. The corresponding field $\underline{E}$, which in the quantum theory becomes the operator of variational derivative with respect to $\underline{A}$, has thus a constant norm along each disjoint component of $\Sigma$. It thus makes sense to replace the $E$ field by a collection of Lie algebra elements $X_{w}$ constant on each wedge. Moreover, as we shall see, the integration over group elements $g_{\epsilon}$ in (22) renders all $X_{w}$ to have the same norm in each disjoint component of $\mathcal{M}$, which is in agreement with what we get in the canonical approach.

The group elements $g_{\epsilon}$ in (22) are "shared" by two dual faces. Thus, all integrals over $g_{\epsilon}$ in (22) have as the integrand the product of two matrix elements of $g_{\epsilon}$. These can be taken using the formula (142) of the Appendix. 


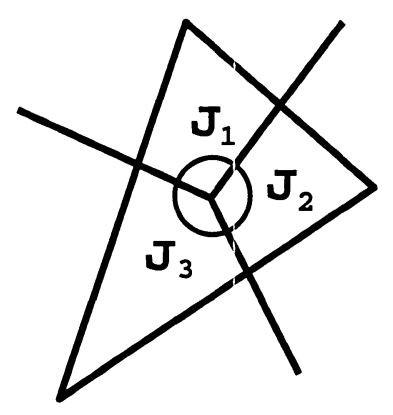

Figure 6: In two dimensions each face is a union of three wedges, and $J_{1}, J_{2}, J_{3}$ are the currents $J_{w}$ corresponding to these wedges.

To describe the result of this integration we will assume that $\mathcal{M}$ has a single connected component. Then the generating functional in two dimensions is given by the following expression

$$
Z_{2}(J, \Delta)=\sum_{j}\left(\operatorname{dim}_{j}\right)^{V-E} \prod_{f} P\left(J_{1}\right) P\left(J_{2}\right) P\left(J_{3}\right)
$$

In the integration over the group elements $g_{\epsilon}$ in (22) survive only the terms in which all spins $j_{\sigma}$ are equal. This spin is denoted by $j$ in (25). The symbols $V, E$ stand in (25) for number of vertices and number of edges in the triangulation correspondingly. The product here is taken over all centers of faces of $\Delta$, or, equivalently, over faces $f$. The graphical notation stands for

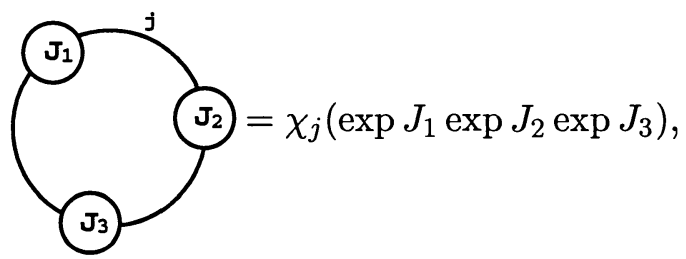

where $J_{1}, J_{2}, J_{3}$ are the three Lie algebra elements (currents) "shared" by the face $f$. Recall that in two dimensions each face is just a union of three wedges, and $J_{1}, J_{2}, J_{3}$ are the currents $J_{w}$ corresponding to these wedges (see Fig. 6). The expression (25) is the final result for the generating functional in two dimensions.

Note that the graph in this graphical representation of the character can be obtained as a result of the following simple construction. Let us draw a circle $S^{1}$ centered at the center of the face (see Fig. 6). The intersection of 


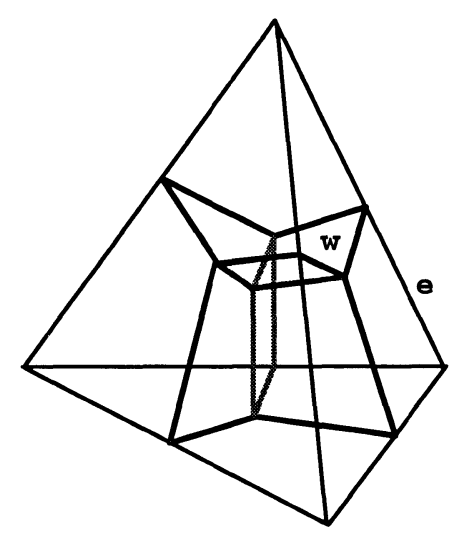

Figure 7: Tetrahedron from a triangulation of a 3-dimensional spacetime manifold. The figure also shows the wedges that lie inside this tetrahedron.

wedges with the circle gives the circle itself. The intersection of this circle with the dual edges gives three points on the circle. Each wedge is labelled by a spin $j$, which is the same for all wedges, and we can assign a label $j$ to the circle that intersects the three wedges. Then the face contribution to (25), that is, the contribution that we graphically represent by $(2.2)$, is simply the spin network constructed with the labelled circle, with insertion of the three group elements $\left(\exp J_{1} \exp J_{2} \exp J_{3}\right)$ at the points where the circle is intersected by the corresponding wedges. This construction is trivial in two dimensions, but turns out to be generalizable to any dimension.

\subsection{3 dimensions}

The three-dimensional case is more interesting. Here the dual faces no longer cover the spacetime manifold, and the $E$ field acquires a true distributional character. The general construction prescribes to replace the $E$ field, which is now a one-form, by a distribution concentrated along dual faces and constant along the wedges. It is illustrative to give a coordinate expression for such a distributional field. Let us consider an arbitrary wedge $w$ (see Fig. 7). Let $u=0$ be the equation of a plane containing $w$. Then the field $E$ that has the correct distributional character is given by the expression

$$
d u \delta(u) X_{w} .
$$

To calculate the generating functional let us note that each edge of the dual triangulation belongs to three dual faces. Thus, in (22), one has to 
take integrals over products of three matrix elements. The corresponding formula is given in the Appendix C. The result of this integration can be described as a generalization of the "circle" construction given at the end of the previous subsection. Let us draw a sphere $S^{2}$ centered at the center of each tetrahedron. The wedges belonging to a particular tetrahedron intersect the sphere and draw a graph on its surface. We will refer to this graph as $\Gamma_{t}$, where $t$ refers to a tetrahedron of the triangulation that was used to construct $\Gamma_{t}$. It is not hard to see that $\Gamma_{t}$ is a tetrahedron whose vertices come from the intersection of the sphere with the dual edges. The same graph $\Gamma_{t}$ can also be obtained by looking at the boundary of tetrahedron $t$ (this boundary has the topology of $S^{2}$ ), which is triangulated by the faces of $t$, and constructing the graph dual to the triangulation of this $S^{2}$. The resulting tetrahedron is the same as that in the previous construction with a sphere.

In (22) the sum is taken over spins $j_{\sigma}$ labelling the dual faces. The result of the integration over the group elements $g_{\epsilon}$ will still be a sum over spins $j_{\sigma}$. Each wedge belongs to some dual face, and, thus, is labelled by spin. As we have just seen the edges of $\Gamma_{t}$ are in one-to-one correspondence with the wedges. Let us label these edges with the same spins as those of the corresponding wedges. We have a current $J_{w}$ associated with each of the 6 wedges belonging to tetrahedron $t$. Let us denote this currents by $J_{1}, \ldots, J_{6}$. We can now construct a function of the group elements $\exp J_{1}, \ldots, \exp J_{6}$ that is just a spin network function.

The generating functional in three dimensions is then given by the following expression

$$
Z_{3}(J, \Delta)=\sum_{j_{\sigma}} \prod_{\sigma} \operatorname{dim}_{j_{\sigma}} \prod_{t} P\left(J_{1}\right) \cdots P\left(J_{6}\right)
$$

where the sum is over all possible coloring of dual faces. This is the final expression we are going to use in the following section in order to derive spin foam models. 


\subsection{4 dimensions}

The case of four dimensions is analogous to the just analyzed case of three dimensions. The only difference is that it is harder to visualize a fourdimensional triangulated manifold, and that the final expression for the generating functional is more complicated. However, the final result for the generating functional follows the same pattern.

First, let us give a coordinate expression for the distributional field $E$ in four dimensions. Let $u, v$ be two functions such that $u=0, v=0$ is the equation for a 2-surface in $\mathcal{M}$ containing one of the faces $\sigma$ of the dual complex, and $d u \wedge d v$ is positive as defined by the orientation of $\sigma$. We then define $E$ to be equal to

$$
d u \wedge d v \delta(u) \delta(v) X_{w}
$$

on each wedge $w$, and to be equal to zero everywhere else. We repeat this construction for all wedges, and add all these distributional forms together to get a two-form that is concentrated along the faces of the dual complex. Then the integral of such distributional $E$ over a wedge of the triangulation is equal simply to $X_{w}$ corresponding to that wedge.

Let us now describe the result of integration over the group elements $g_{\epsilon}$ in (22). In four dimensions each dual edge is shared by four dual faces. Thus, one has to take the integral of the product of four matrix elements. The required formula is given in Appendix C. The result of this integration can again be described using a certain spin network function of the group elements corresponding to the currents $J_{w}$. As in the case of two and three dimensions, let us introduce special graphs in the vicinity of the center of each 4-simplex $h$, which we will call $\Gamma_{h}$. We define graph $\Gamma_{h}$ as the intersection of the sphere $S^{3}$ surrounding the center of 4 -simplex $h$ with the dual faces $\sigma$. The graph $\Gamma_{h}$ lives in $S^{3}$, but it can be projected on a plane. This gives us the pentagon graph. Note that at this stage we do not care about types of crossings we get (that is, whether this is under- or over-crossing). The edges of this graph are in one-to-one correspondence with the wedges $w$ belonging to the 4 -simplex $h$. Thus, we can associate to each edge a current $J_{w}$, and label it with the spin $j_{\sigma}$ labelling the dual face to which the corresponding wedge belongs. Additionally, let us label each of five vertices of the pentagon by a half-integer (spin). Vertices of the pentagon are in one-to-one correspondence with tetrahedra of the triangulation. Thus, we shall use the notation $j_{t}$ for this spins.

Inside each four-simplex there are 10 wedges. Let us denote the corresponding currents by $J_{1}, \ldots, J_{10}$. One can then construct the function of the group elements $\exp J_{1}, \ldots, \exp J_{10}$. 
The generating functional $Z_{4}$ is given by the sum over spins of products over 4-simplices of the above functions of the currents $J_{w}$

$$
Z_{4}(J, \Delta)=\sum_{j_{\sigma}, j_{t}} \prod_{\sigma} \operatorname{dim}_{j_{\sigma}} \prod_{t} \operatorname{dim}_{j_{t}} \prod_{h} P\left(J_{1}\right) \cdots P\left(J_{10}\right)
$$

Here $h$ labels 4-simplices of the triangulation and $j_{\sigma}$ are spins labelling the dual faces. The resolution of each vertex in this pentagon graph is given in the Appendix C.

\section{Theories of interest}

Having calculated the generating functional in various spacetime dimensions we are in the position to use our results to study concrete theories. However, let us first specify in more details the class of theories that can be treated within our approach, and give physically interesting examples of theories belonging to this class. In this paper we will restrict our attention to a special class of theories. For simplicity we fix the gauge group to be $G=\mathrm{SU}(2)$. Let $\mathcal{M}$ be an oriented smooth d-dimensional manifold, and let $P$ be an SU(2)bundle over $\mathcal{M}$. The basic fields of a theory of interest are a connection $A$ on $P$, and an $\operatorname{Ad} P$-valued (d-2)-form, often called $B$, which we call $E$ because of its relation with the $\mathrm{SU}(2)$ "electric" field of the canonical formulation of the theory. The action of the theory is of the form

$$
S[A, E]=\int_{\mathcal{M}}[\operatorname{Tr}(E \wedge F)+\Phi(E)]
$$

where $F$ is the curvature of $A$, 'Tr' is the trace in the fundamental representation of $\mathrm{SU}(2)$, and $\Phi$ is a gauge invariant polynomial function that depends on $E$ but not on its derivatives. Also, $\Phi(E)$ should be a d-form so that it can be integrated. It can also depend on other dynamical fields, for example Lagrange multipliers as in the case of gravity, or on additional non-dynamical fields (background structure), as in the case of Yang-Mills theories. Let us now give examples of theories belonging to this class. 


\subsection{BF theories}

The action of BF theory in any dimension is given by

$$
\int_{\mathcal{M}} \operatorname{Tr}(E \wedge F)
$$

that is, this is the action of the type (31) with zero "interaction" term $\Phi$. The equations of motion that follow from this action state that

$$
d_{A} E=0, \quad F=0,
$$

where $d_{A} E$ is the covariant derivative of $E$. Thus, the flat connections play the dominant role in $\mathrm{BF}$ theory.

\subsection{D Yang-Mills}

Yang-Mills action can be written only if one introduces a background metric on $\mathcal{M}$. However, in two spacetime dimensions, Yang-Mills action turns out to depend only on a measure (area two-form) defined by the metric on $\mathcal{M}$. Thus, we will keep track only of this dependence of the action on a measure $d \mu$ on $\mathcal{M}$. Yang-Mills theory in 2D is described by the following BF-like action

$$
\int_{\mathcal{M}} \operatorname{Tr}(E F)+\frac{e^{2}}{2} \int_{\mathcal{M}} d \mu \operatorname{Tr}\left(E^{2}\right)
$$

Here $E$ is a Lie-algebra valued zero-form, $F$ is the curvature of the connection $A$. Solving equations of motion for $E$ that follow from this action, and substituting the solution into the action, one can check that the action reduces to the standard Yang-Mills action

$$
S_{\mathrm{YM}}=-\frac{1}{4 e^{2}} \int_{\mathcal{M}} d^{2} x \sqrt{g} g^{a c} g^{b d} \operatorname{Tr}\left(F_{a b} F_{c d}\right),
$$

where $g_{a b}$ is a metric (of Euclidean signature) on $\mathcal{M}$ such that $d^{2} x \sqrt{g}=d \mu$, where $\sqrt{g}$ is the square root of the determinant of the metric.

Using the techniques developed in this paper, one can calculate the vacuum-vacuum transition amplitude of this theory, that is the path integral of $\exp (i \times$ action$)$. However, an interesting feature of this theory is that the same techniques can be used to calculate the partition function, that is, the path integral

$$
\int \mathcal{D} A \mathcal{D} E \exp \left(-S_{\mathrm{YM}}\right)
$$


The problem of calculation of the partition function can be reduced to the problem of calculation of the vacuum-vacuum transition amplitude of a somewhat different theory, that is of the path integral of $\exp (i \times$ action $)$, with the action given by

$$
\int_{\mathcal{M}} \operatorname{Tr}(E F)-i \frac{e^{2}}{2} \int_{\mathcal{M}} d \mu \operatorname{Tr}\left(E^{2}\right) .
$$

Indeed, integrating over $E$ in the path integral of $\exp (i \times$ action $)$, one gets (36). As one can see, the two actions (34),(37) differ by the factor of $i$ in front of the second term. Using the two actions (34),(37) we will calculate below both the vacuum-vacuum transition amplitude and partition function of the theory.

\subsection{D BF theory with the 'cosmological term'}

In three dimensions the $E$ field of BF theory is a one-form, and one can add a term cubic in $E$ to the $\mathrm{BF}$ action to obtain

$$
-\int_{\mathcal{M}} \operatorname{Tr}\left(E \wedge F+\frac{\Lambda}{12} E \wedge E \wedge E\right),
$$

where $\mathcal{M}$ is assumed to be a three-dimensional orientable manifold. The action is a functional of an $\mathrm{SU}(2)$ connection $A$, whose curvature form is denoted by $F$, and a 1 -form $E$, which takes values in the Lie algebra of $\mathrm{SU}(2)$. Thus, the action (38) is that of BF theory in 3d, with $E$ field playing the role of $B$, and with an additional "cosmological term" added to the usual $\mathrm{BF}$ action. This theory is related to gravity in 3D as follows. Having the one-form $E$, one can construct from it a real metric of Euclidean signature

$$
g_{a b}=-\frac{1}{2} \operatorname{Tr}\left(E_{a} E_{b}\right) .
$$

Here $a, b$ stand for spacetime indices: $a, b, \ldots=1,2,3$. We take the $E$ field to be anti-hermitian, which explains the minus sign in (39). Thus, the $E$ field in (38) has the interpretation of the triad field. One of the equations of motion that follows from (38) states that $A$ is the spin connection compatible with the triad $E$. Taking the $E$ field to be non-degenerate and "right-handed", i.e., giving a positive-definite volume form

$$
\frac{1}{12} \tilde{\varepsilon}^{a b c} \operatorname{Tr}\left(E_{a} E_{b} E_{c}\right),
$$

and substituting into (38) the spin connection instead of $A$, one gets the Euclidean Einstein-Hilbert action

$$
\frac{1}{2} \int_{\mathcal{M}} d^{3} x \sqrt{g}(R-2 \Lambda) .
$$


We use units in which $8 \pi G=1$. The minus in front of (38) is needed to yield precisely the Einstein-Hilbert action after the elimination of $A$. Thus, $\Lambda$ in (38) is the cosmological constant. An important difference of this theory and Einstein's theory is the fact BF theory, unlike gravity, is defined for degenerate metrics.

\subsection{D BF theory with the 'cosmological term'}

In four spacetime dimensions the $E$ field of BF theory is a two-form. Thus, one can construct the following action functional

$$
\int_{\mathcal{M}} \operatorname{Tr}(E \wedge F)-\frac{\Lambda}{2} \operatorname{Tr}(E \wedge E),
$$

where $\Lambda$ is a real parameter, which we will refer to as the "cosmological constant". If one, as in the following subsection, adds to this action an additional constraint that $E$ is simple, that is, given by a product of two one-forms, then this theory is equivalent to Einstein's theory and $\Lambda$ is proportional to the "physical" cosmological constant.

\subsection{D Euclidean self-dual gravity}

The action for Euclidean general relativity in the self-dual first order formalism is given by [24]

$$
\int_{\mathcal{M}} \operatorname{Tr}(E \wedge F)-\psi^{i j}\left(E^{i} \wedge E^{j}-\frac{1}{3} \delta^{i j} E^{k} \wedge E_{k}\right),
$$

where, to write $E^{i}$, we have introduced a basis in the Lie algebra of SU(2). Here $\psi^{i j}$ is a symmetric matrix of Lagrange multipliers. The variation of the action with respect to $\psi$ yields equations

$$
E^{i} \wedge E^{j}=\delta^{i j} \frac{1}{3} E^{k} \wedge E_{k}
$$

In the case $E$ is non degenerate, i.e., the right hand side of (44) is non zero, these equations are satisfied if and only if $E$ is the self-dual part of a decomposable 2-form, i.e., if and only if there exists a tetrad field $e^{I}, I=$ $0,1,2,3$ such that $E^{i}= \pm\left(e^{0} \wedge e^{i}+\frac{1}{2} \epsilon_{i j k} e^{j} \wedge e^{k}\right)$. In this case the action reduces to the self-dual Hilbert-Palatini action for 4-dimensional gravity and $\psi_{i j}$ correspond to the components of the self-dual part of the Weyl curvature tensor. Thus, (43) is the BF action in four dimensions, with an additional "simplicity" constraint added to it. 


\section{Corresponding spin foam models}

In this section we present state sum models for the theories we just described.

\subsection{Ponzano-Regge-Ooguri models}

These models give an expression for the vacuum-vacuum transition amplitude of BF theory, i.e., the path integral

$$
Z^{\mathrm{BF}}(\mathcal{M})=\int \mathcal{D} A \mathcal{D} E \exp \left(i \int_{\mathcal{M}} \operatorname{Tr}(E \wedge F)\right)
$$

as a sum over colored triangulations. More precisely, let us fix a triangulation $\Delta$ of $\mathcal{M}$. In three dimensions, let us label the edges of $\Delta$ by irreducible representations of $\mathrm{SU}(2)$, that is, by spins $j$. Thus, to each edge $e$ we assign a label $j_{e}$. One can then construct the following sum over labellings

$$
\operatorname{PR}(\Delta)=\sum_{j_{e}} \prod_{e} \operatorname{dim}_{j_{e}} \prod_{t}(6 j)_{t}
$$

Here $\operatorname{dim}(j)=2 j+1$ is the dimension of the representation $j$, the second product is taken over tetrahedra $t$ of $\Delta$, and $(6 j)$ is the (normalized) classical (6j)-symbol (see the Appendix $\mathrm{C}$ for a definition) constructed from the six spins labelling the edges of $t$. Note that the sum over spins in (46) diverges. To make sense of it one must introduce a regularization. A possible regularization is given by the Turaev-Viro model, which we discuss below. After the introduction of this "regularization", this sum can be shown to be triangulation independent. Thus, (46) gives an invariant of $\mathcal{M}: \operatorname{PR}(\Delta)=\operatorname{PR}(\mathcal{M})$.

In four dimensions one can construct a similar sum by labelling the faces of $\Delta$. Let us denote the spin labelling a face $f$ by $j_{f}$. Let us consider the following quantity:

$$
\mathrm{O}(\Delta)=\sum_{j_{f}, j_{t}} \prod_{f} \operatorname{dim}_{j_{f}} \prod_{t} \operatorname{dim}_{j_{t}} \prod_{h}(15 j)_{h}
$$

After a certain "regularization" (given by the Crane-Yetter model below) that gives sense to the infinite sums in (47), this can be shown to be triangulation independent: $\mathrm{O}(\Delta)=\mathrm{O}(\mathcal{M})$. In (47) one has introduced an additional label $j_{t}$ for each tetrahedron of $\Delta$. The spin $j_{t}$ labels an intertwiner one has to assign to each tetrahedron (see the subsection on Crane-Yetter model for details). The last product is taken over the 4-simplices $h$ of $\Delta$. Then $(15 j)$ is the (normalized) $(15 \mathrm{j})$-symbol constructed from ten spins $j_{f}$ labelling the faces of $h$ and five spins $j_{t}$ labelling the five tetrahedra composing $h$. See the Appendix $\mathrm{C}$ for a definition of the normalized (15j)-symbol. 
As was realized by Ooguri [9], these models give a "discrete" realization of the path integral because they can be obtained from the requirement that the connection on $\mathcal{M}$ is flat. Indeed, formally the path integral (45) is equal to

$$
\int \mathcal{D} A \delta(F)
$$

The state sums (46),(47) are in certain precise sense realizations of the integral over connections with the integrand being the delta-function at $F=0$.

\subsection{Migdal-Witten model}

Migdal [5] has studied the Yang-Mills theory on a lattice, and, in particular, has proposed a lattice model for the Yang-Mills theory in two dimensions. This model was later studied by Witten [6] in the connection with topological field theories in two dimensions.

As we discussed above, the problem of calculation of the Yang-Mills partition function can be reduced to the problem of calculation of the path integral

$$
Z^{\mathrm{YM}}\left(\rho e^{2}, \mathcal{M}\right)=\int \mathcal{D} A \mathcal{D} E \exp \left(i \int_{\mathcal{M}} \operatorname{Tr}(E F)+\frac{e^{2}}{2} \int_{\mathcal{M}} d \mu \operatorname{Tr}\left(E^{2}\right)\right)
$$

where $\rho$ is the total area of $\mathcal{M}$, and other notations are explained in subsection (3.2). Migdal [5] has proposed the following lattice version of this path integral. For simplicity, we will formulate Migdal model on a triangulated manifold. Let us triangulate our two-dimensional manifold $\mathcal{M}$, and introduce the dual triangulation (see Fig. 5). Let us label the edges $\epsilon$ of the dual complex by group elements $g_{\epsilon}$, and the dual faces $\sigma$ by irreducible representations of SU(2), i.e., spins $j_{\sigma}$. The "discrete" version of (49) is then given by (see [6]):

$$
\mathrm{YM}\left(\rho e^{2}, \mathcal{M}\right)=\prod_{\epsilon} \int d g_{\epsilon} \sum_{j_{\sigma}} \prod_{\sigma} \operatorname{dim}_{j_{\sigma}} \chi_{j_{\sigma}}\left(g_{\epsilon_{1}} \cdots g_{\epsilon_{n}}\right) \exp \left(-e^{2} \rho_{\sigma} c\left(j_{\sigma}\right) / 2\right),
$$

where the multiple integral is performed over all group elements $g_{\epsilon}$ and $d g$ is the normalized Haar measure on $\mathrm{SU}(2), \rho_{\sigma}$ is the area of the dual face $\sigma$, as defined by the measure $d \mu, c(j)$ is the value of the quadratic Casimir operator in the representation $j$

$$
c(j)=2 j(j+1)
$$

and $g_{\epsilon_{1}} \cdots g_{\epsilon_{n}}$ is the product of group elements around the dual face. After integration over the group elements $g_{\epsilon}$ the partition function takes the 
following simple form:

$$
\sum_{j}\left(\operatorname{dim}_{j}\right)^{\kappa(\mathcal{M})} e^{-e^{2} \rho c(j)}
$$

where $\kappa(\mathcal{M})$ is the Euler characteristics of $\mathcal{M}$. Thus, as we indicated in the argument of $\mathrm{YM}$, the partition function depends only on the topological properties of $\mathcal{M}$. Also, the dependence on measure $d \mu$ enters only through the dependence on the total area $\rho$ of $\mathcal{M}$.

\subsection{Turaev-Viro model}

The Turaev-Viro model gives a way to calculate the transition amplitude of the theory defined by (38), i.e., the path integral

$$
Z_{3}^{\mathrm{BF}}(\Lambda, \mathcal{M})=\int \mathcal{D} E \mathcal{D} A \exp \left(-i \int_{\mathcal{M}} \operatorname{Tr}\left(E \wedge F+\frac{\Lambda}{12} E \wedge E \wedge E\right)\right) .
$$

In this paper we consider only vacuum-vacuum amplitudes. Although the Turaev-Viro model can be used to calculate more general amplitudes between non-trivial initial and final states, we will not use this aspect of the model here. We consider the version of the model formulated on a triangulated manifold. Thus, let us fix a triangulation $\Delta$ of $\mathcal{M}$. Let us label the edges, for which we will employ the notation $e$, by irreducible representations of the quantum group $(\mathrm{SU}(2))_{q}$, where $q$ is a root of unity

$$
q=e^{\frac{2 \pi i}{k}} \equiv e^{i \hbar}
$$

Later we will relate the parameter $\hbar$ with the cosmological constant $\Lambda$. The irreducible representations of $(\mathrm{SU}(2))_{q}$ are labelled by half-integers (spins) $j$ satisfying $j \leq(k-2) / 2$. Thus, we associate a spin $j_{e}$ to each edge $e$. The vacuum-vacuum transition amplitude of the theory is then given by the following expression (see, for example, [25]):

$$
\operatorname{TV}(q, \Delta)=\eta^{2 V} \sum_{j_{e}} \prod_{e} \operatorname{dim}_{q}\left(j_{e}\right) \prod_{t}(6 j)_{q}
$$

where $\eta$ and the so-called quantum dimension $\operatorname{dim}_{q}(j)$ are defined in the Appendix A by (129) and (130) correspondingly, and $V$ is the number of vertices in $\Delta$. The last product in (55) is taken over tetrahedra $t$ of $\Delta$, and $(6 j)_{q}$ is the (normalized) quantum $(6 j)$-symbol constructed from the 6 spins labelling the edges of $t$ (see Appendix C). It turns out that (55) is independent of the triangulation $\Delta$ and gives a topological invariant of $\mathcal{M}$ : $\operatorname{TV}(q, \Delta)=\operatorname{TV}(q, \mathcal{M})$. 
The construction that interprets the Turaev-Viro invariant (55) as the vacuum-vacuum transition amplitude of the theory defined by (38) is as follows. It has been proved (see e.g. [25]) that (55) is equal to the squared absolute value of the Chern-Simons amplitude

$$
\operatorname{TV}(q, \mathcal{M})=|\operatorname{CS}(k, \mathcal{M})|^{2},
$$

with the level of Chern-Simons theory being equal to $k$ from (54). It is known, however, that the action (38) can be written as a difference of two copies of Chern-Simons action

$$
S_{\mathrm{CS}}(A)=\frac{k}{4 \pi} \int_{\mathcal{M}} \operatorname{Tr}\left(A \wedge d A+\frac{2}{3} A \wedge A \wedge A\right) .
$$

Indeed, note that

$$
S(A+\lambda E)-S(A-\lambda E)=\frac{k \lambda}{\pi} \int_{\mathcal{M}} \operatorname{Tr}\left(E \wedge F+\frac{\lambda^{2}}{3} E \wedge E \wedge E\right),
$$

where $\lambda$ is a real parameter. Thus, (58) is equal to (38) if

$$
\lambda=-\sqrt{\Lambda} / 2, \quad k=\frac{2 \pi}{\sqrt{\Lambda}}, \quad \text { or } \quad \hbar=\sqrt{\Lambda} .
$$

This relates the deformation parameter $q$ of the Turaev-Viro model with the cosmological constant $\Lambda$, and proves that the Turaev-Viro amplitude is proportional to the vacuum-vacuum transition amplitude of the theory defined by (38)

$$
\mathrm{TV}(q, \mathcal{M}) \propto Z_{3}^{\mathrm{BF}}(\Lambda, \mathcal{M})
$$

To compare in Sec. 5 the spin foam model obtained via our techniques with the Turaev-Viro model, we will need the first-order term in the decomposition of (55) in the power series in $\Lambda$. This is proportional to the expectation value of the spacetime volume in BF theory at $\Lambda=0$. Indeed, the expectation value of the volume is given simply by the derivative of the amplitude (6) with respect to $(-i \Lambda)$ evaluated at $\Lambda=0$ :

$$
\begin{aligned}
\langle\operatorname{Vol}\rangle=\frac{\int \mathcal{D} E \mathcal{D} A \operatorname{Vol}(\mathcal{M}) e^{i S}}{\int \mathcal{D} E \mathcal{D} A e^{i S}} & =i\left(\frac{\partial \ln Z_{3}^{\mathrm{BF}}(\Lambda)}{\partial \Lambda}\right)_{\Lambda=0}, \\
\operatorname{Vol}(\mathcal{M}) & =\int_{\mathcal{M}} \frac{1}{12} \tilde{\varepsilon}^{a b c} \operatorname{Tr}\left(E_{a} E_{b} E_{c}\right) .
\end{aligned}
$$

Thus, the expectation value of the volume of $\mathcal{M}$ in BF theory is proportional to the first order term in $\Lambda$ in the decomposition of (55). 
Let us find this expectation value. An important subtlety arises here. It is not hard to show that (55) has the following asymptotic expansion in $\hbar$

$$
\left(\frac{\hbar^{3}}{4 \pi}\right)^{V} \operatorname{PR}(\Delta)\left(1-\hbar^{2} i\langle\mathrm{Vol}\rangle\right),
$$

where PR is the amplitude of the Ponzano-Regge model (46) $V$ is the number of vertices in $\Delta$, and $i\langle\mathrm{Vol}\rangle$ is a real quantity independent of $\hbar$. Thus, apparently there is no term proportional to $\hbar^{2}=\Lambda$ in this expansion. However, as it is explained, for example, in [26], the state sum invariant (55) does not exactly give the transition amplitude of BF theory. Instead, the Turaev-Viro amplitude is only proportional to the $\mathrm{BF}$ amplitude, and the proportionality coefficient depends on $\hbar$. This can be understood as follows. The integration over $(A+\lambda E),(A-\lambda E)$, which is carried out to obtain $|\mathrm{CS}(k, \mathcal{M})|^{2}$ in $(56)$ and thus the Turaev-Viro amplitude, is different from the integration over $A, E$ one has to perform to obtain (53). The difference in the integration measures is a power of $\hbar$. Thus, the amplitude (53) and the squared absolute value of the amplitude of the Chern-Simons theory are proportional to each other with the coefficient of proportionality being a power of $\hbar$. In the discretized version of the theory, given by the Turaev-Viro model, this power of $\hbar$ is replaced by $\hbar^{3 V}$. Thus, the Turaev-Viro amplitude, in the limit of small cosmological constant, differs from the BF amplitude by a power of $\hbar^{3 V}$.

This remark being made, we can write an expression for the expectation value of the volume in BF theory:

$$
\begin{aligned}
& i\langle\mathrm{Vol}\rangle_{\Delta}=-\frac{\partial}{\partial \Lambda}\left(\frac{\mathrm{TV}(\Lambda, \Delta)}{\operatorname{PR}(\Delta)\left(\hbar^{3} / 4 \pi\right)^{V}}\right)_{\Lambda=0}= \\
& \frac{1}{\operatorname{PR}(\Delta)} \sum_{j_{e}} i \operatorname{Vol}(\Delta, \mathrm{j})\left(\prod_{e} \operatorname{dim}\left(j_{e}\right) \prod_{t}(6 j)\right)
\end{aligned}
$$

where the function $\operatorname{Vol}(\Delta, \mathbf{j})$ of the triangulation $\Delta$ and the labels $\mathbf{j}=\left\{j_{e}\right\}$ is given by

$$
\begin{aligned}
i \operatorname{Vol}(\Delta, \mathbf{j})= & \sum_{v}\left(-\frac{\partial}{\partial \Lambda}\left(\frac{\eta^{2}}{\left(\hbar^{3} / 4 \pi\right)}\right)\right)_{\Lambda=0}+\sum_{e}\left(-\frac{\partial \ln \left(\operatorname{dim}_{q}\left(j_{e}\right)\right)}{\partial \Lambda}\right)_{\Lambda=0} \\
& +\sum_{t}\left(-\frac{\partial \ln \left((6 j)_{q}\right)}{\partial \Lambda}\right)_{\Lambda=0} .
\end{aligned}
$$

Here $v$ stands for vertices of $\Delta, e$ stands for edges and $t$ stands for tetrahedra. We intentionally wrote the expectation value of the volume in the form (63) 
to introduce the volume $\operatorname{Vol}(\Delta, \mathbf{j})$ of a labelled triangulation, which will be of interest to us in what follows. Indeed, (63) has the form

$$
\frac{\sum_{j_{e}} i \operatorname{Vol}(\Delta, \mathbf{j}) \operatorname{Amplitude}(\Delta, \mathbf{j})}{\sum_{j_{e}} \operatorname{Amplitude}(\Delta, \mathbf{j})},
$$

where

$$
\operatorname{Amplitude}(\Delta, \mathbf{j})=\prod_{e} \operatorname{dim}\left(j_{e}\right) \prod_{t}(6 j)
$$

is the amplitude of Ponzano-Regge model. This shows that $\operatorname{Vol}(\Delta, \mathbf{j})$ indeed has the interpretation of the volume of a labelled triangulation. Note that the volume turns out to be purely imaginary. This has to do with the fact that in (53) one sums over configurations of $E$ of both positive and negative volume. A more detailed explanation of this is given in [26].

The volume (64) has three types of contributions: (i) from vertices; (ii) from edges; (iii) from tetrahedra. It is not hard to calculate the first two types of them. One finds that each vertex contributes exactly $1 / 12$, and each edge contributes $j_{e}\left(j_{e}+1\right) / 6$, where $j_{e}$ is the spin that labels the edge $e$. It is much more complicated to find the tetrahedron contribution to the volume, that is, the derivative of $\ln \left((6 j)_{q}\right)$ with respect to $\Lambda$. The result is described in the Appendix D. Using the notations of the Appendix D, the final result for the expectation value of the spacetime volume in BF theory can be written as follows:

$$
\begin{aligned}
& i \operatorname{Vol}(\Delta, \mathbf{j})=\sum_{v} \frac{1}{12}+\sum_{e} \frac{j_{e}\left(j_{e}+1\right)}{6}+\sum_{t} \frac{1}{16} \frac{1}{\vartheta} \times
\end{aligned}
$$

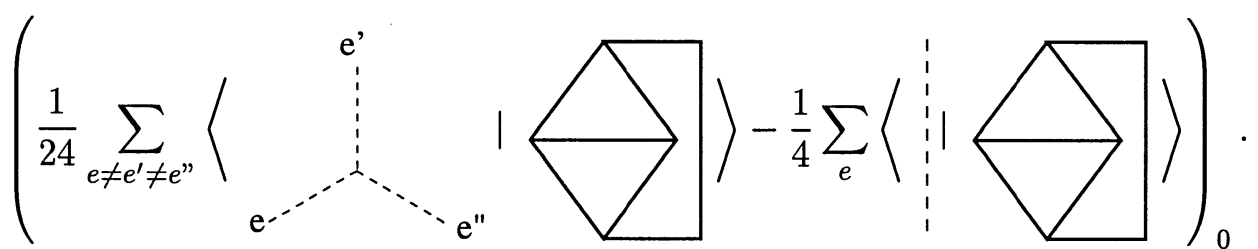

Here $\vartheta$ stands for the normalized classical $(6 j)$-symbol.

It is interesting to note that not only tetrahedra $t$ of $\Delta$ contribute to the volume, but also the edges $e$ and vertices $v$. The contribution from the vertices is somewhat trivial - it is constant for each vertex. Nevertheless, when thinking about the triangulated manifold $\mathcal{M}$ one is forced to assign the spacetime volume to every vertex. The contribution from edges depends on the spins labelling the edges. Again, this implies that each edge of the triangulation $\Delta$ carries an intrinsic volume that depends on its spin. The contribution from tetrahedra is more complicated. It is given by a function that depends on the spins labelling the edges of each tetrahedron. It is 
interesting that this picture of the spacetime volume being split into contributions from vertices, edges and tetrahedra can be understood in terms of Heegard splitting of $\mathcal{M}$. Recall that Heegard splitting of a three-dimensional manifold $\mathcal{M}$ decomposes $\mathcal{M}$ into three dimensional manifolds with boundaries. Then the original manifold can be obtained by gluing these manifolds along the boundaries. For the case of a triangulated manifold $\mathcal{M}$, as we have now, the Heegard splitting proceeds as follows. First, one constructs balls centered at the vertices of $\Delta$. Then one connects these balls with cylinders, whose axes of cylindrical symmetry coincide with the edges of $\Delta$. Removing from $\mathcal{M}$ the obtained balls and cylinders, one obtains a three-dimensional manifold with a complicated boundary. One has to further cut this manifold along the faces of $\Delta$. One obtains three types of "building blocks" that are needed to reconstruct the original manifold: (i) balls; (ii) cylinders; (iii) spheres with four discs removed. Each of this manifolds carries a part of the original volume of $\mathcal{M}$. Our result (67) provides one with exactly the same picture: the volume of $\mathcal{M}$ is concentrated in vertices (balls of the Heegard splitting), edges (cylinders), and tetrahedra (4-holed spheres).

\subsection{Crane-Yetter model}

Crane, Kauffman and Yetter [10] studied a state sum model that is very similar to the Ooguri model [9], however, instead of the gauge group $\mathrm{SU}(2)$ the quantum group $(\mathrm{SU}(2))_{q}$ is used. The state sum invariant they proposed is given by (see, for example, [25]):

$$
\mathrm{CY}(q, \Delta)=\eta^{-2 V+2 E} \sum_{j_{f}} \sum_{j_{t}} \prod_{f} \operatorname{dim}_{q}\left(j_{f}\right) \prod_{t} \operatorname{dim}_{q}\left(j_{t}\right) \prod_{h}(15 j)_{q} .
$$

Here $\operatorname{dim}_{q}(j)$ is the quantum dimension (130), $\eta$ is defined by (129), $(15 j)_{q}$ stands for a (normalized) quantum (15j)-symbol (see the Appendix C) associate with any 4-simplex. This quantum (15j)-symbol is the Reshetikhin Turaev evaluation of the graph $\Gamma_{h}$ described in subsection (2.4). The symbols $V$ and $E$ stand for the number of vertices and edges in $\Delta$ correspondingly. This state sum model is independent of a triangulation of the 4-manifold used to compute (68). Thus, it gives an invariant associated with the manifold $\mathcal{M}: \mathrm{CY}(q, \Delta)=\mathrm{CY}(q, \mathcal{M})$.

The quantity $\mathrm{CY}(q, \mathcal{M})$ is conjectured to give the transition amplitude for $B F$ theory with cosmological constant, the cosmological constant $\Lambda$ being related to $q$ by

$$
q=\exp i \Lambda \text {. }
$$

Thus, unlike in the case of 3-dimensions, we have now $\hbar=\Lambda$. This relation can be established as follows. It has been shown (see e.g. [25]) that if $\mathcal{M}$ is a 
manifold with boundary then $\mathrm{CY}(q, \mathcal{M})$ is proportional to the Chern-Simons transition amplitude $\operatorname{CS}(k, \partial M)$ introduced in the previous subsection:

$$
\mathrm{CY}(q, \mathcal{M}) \propto \mathrm{CS}(k, \partial \mathcal{M}) .
$$

The deformation parameter $q$ of the Crane-Yetter model is related to the level of Chern-Simons theory as in (54). Consider now the transition amplitude for $B F$ theory with cosmological term:

$$
Z_{4}^{\mathrm{BF}}(\Lambda, \mathcal{M})=\int \mathcal{D} A \mathcal{D} E \exp \left(i \int_{\mathcal{M}} \operatorname{Tr}(E \wedge F)-\frac{\Lambda}{2} \operatorname{Tr}(E \wedge E)\right) .
$$

Let us integrate over the field $E$ in this path integral. One gets

$$
Z_{4}^{\mathrm{BF}}(\Lambda, \mathcal{M}) \propto \int \mathcal{D} A \exp \left(\frac{i}{2 \Lambda} \int_{\mathcal{M}} \operatorname{Tr}(F \wedge F)\right) .
$$

Now, using the fact that

$$
\operatorname{Tr}(F \wedge F)=d\left(A \wedge d A+\frac{2}{3} A \wedge A \wedge A\right)
$$

we get

$$
Z_{4}^{\mathrm{BF}}(\Lambda, \mathcal{M}) \propto \int \mathcal{D} A \exp \left(\frac{i}{2 \Lambda} S_{\mathrm{CS}}(A, \partial \mathcal{M})\right) .
$$

This, together with (70), then implies that

$$
\mathrm{CY}(q, \mathcal{M}) \propto Z_{4}^{\mathrm{BF}}(\Lambda, \mathcal{M})
$$

where $q$ is related to $\Lambda$ as in (69).

To compare the spin foam model we obtain below with the Crane-Yetter model we will need to know the first order term in the decomposition of $Z_{4}^{\mathrm{BF}}(\Lambda, \mathcal{M})$ in the power series in $\Lambda$. To find it, we have to take into account the fact that, similarly to the case of 3D BF theory, the transition amplitude is only proportional to the Crane-Yetter amplitude (68), with the proportionality coefficient being a function of $\hbar$. This proportionality coefficient is given by a power of $\hbar$. As one can check, the "zeroth" order term in $\hbar$ of (68) is equal to

$$
\left(\frac{\hbar^{3}}{4 \pi}\right)^{-V+E} O(\Delta)
$$

where $O(\Delta)$ is the Ooguri state sum invariant (47). Thus, the first order term in $\Lambda$ of $Z_{4}^{\mathrm{BF}}(\Lambda, \mathcal{M})$ is given by

$$
\left(\frac{\partial}{\partial \Lambda} Z_{4}^{\mathrm{BF}}(\Lambda, \mathcal{M})\right)_{\Lambda=0}=\left(\frac{\partial}{\partial \Lambda}\left(\frac{\mathrm{CY}(q, \mathcal{M})}{\left(\hbar^{3} / 4 \pi\right)^{-V+E}}\right)\right)_{\Lambda=0} .
$$


However, unlike the case of 3D BF theory with cosmological term, in this case the only contribution to this expression comes from the quantum (15j)symbol. All other "building blocks" that one encounters in (68) contain only the $\hbar^{2}=\Lambda^{2}$ terms, and, thus, do not contribute to (77). The first derivative of the quantum (15j)-symbol with respect to $\hbar=\Lambda$ can be found by methods analogous to the ones used in the Appendix $\mathrm{D}$ to calculate the derivative of (6j)-symbol. However, in the case of (15j)-symbol, the calculation is much simpler due to the fact that only crossings contribute to the first order in $\hbar$. The (15j)-symbol used in (68) contains only one crossing (see, for example, [25]). Using the expression (152) for the R-matrix as a formal power series in $\hbar$ given in the Appendix $\mathrm{D}$, one can easily check that the first $\hbar$-order term of the quantum (15j)-symbol is given by:

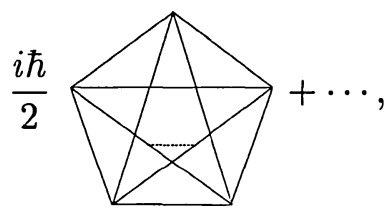

where the dots stands for terms containing graspings between two edges sharing one vertex and correspond to different possible choices of the framing of the $15 \mathrm{j}$-symbol. We will not keep track of these terms at this stage. Their relevance will be discussed below. This is, however, not quite the expression we want because it is not symmetrical. We will symmetrize it by putting the grasping at all pairs of lines of the graph in (78) that do not share a vertex. However, if one does that, than the quantity one obtains for each given 4 -simplex $h$ is not equal to (78). Only if one sums over all 4 -simplices of the triangulation one obtains the quantity that is equal to the sum of (78) over $h$. This can be proved by using certain simple relations that hold for graspings. One such relation is the analogs of "closure" relations (160). It graphical representation is given by:

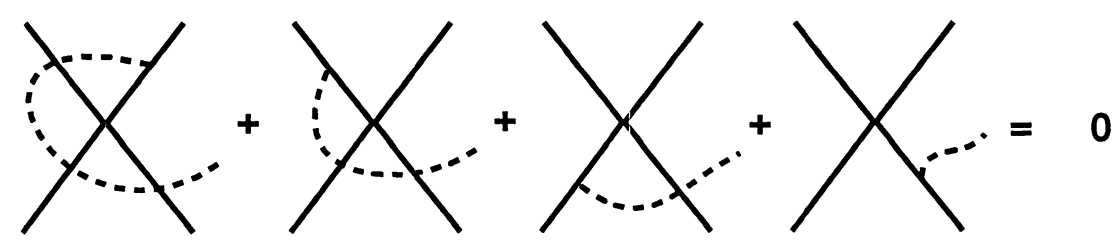

Such "closure" relation holds for every vertex of the graph in (78). Another relation that one has to use involves two different pentagon graphs. As one can convince oneself, the vertices of the pentagon graph in (78) are in oneto-one correspondence with the tetrahedra of $\Delta$. Thus, every tetrahedron is 
shared exactly by two 4-simplices, and the following relations holds:

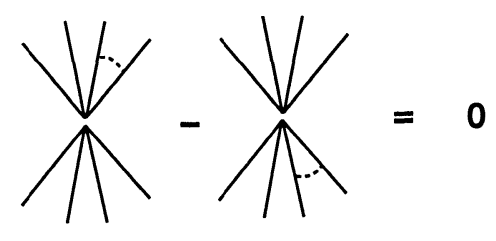

This allows one to cancel certain types of graspings from one 4-simplex with similar types of graspings from the neighboring 4-simplex. Certain cancellations occur for each tetrahedron of the triangulation. These relations allow one to obtain the following symmetric expression:

$$
\begin{aligned}
& \left(\frac{\partial}{\partial \Lambda} Z_{4}^{\mathrm{BF}}(\Lambda, \mathcal{M})\right)_{\Lambda=0}= \\
& \frac{i}{2} \sum_{h} \frac{1}{30}\left(\sum_{e, e^{\prime}} \operatorname{sign}\left(e, e^{\prime}\right)\right\rangle_{0}^{e}
\end{aligned}
$$

where the sum is taken over different pairs $e, e^{\prime}$ of edges that do not share a vertex. There are 30 terms in this sum, which explains the factor of $1 / 30$ in (81). The quantity $\operatorname{sign}\left(e, e^{\prime}\right)$ in (81) is plus or minus one depending on the orientation of the two edges $e, e^{\prime}$. A consistent choice of orientations comes from the geometrical 4-simplex discussed in the Appendix E. With this choice, $\operatorname{sign}\left(e, e^{\prime}\right)=\operatorname{sign}\left(f, f^{\prime}\right)$, where we use the fact that every edge of the pentagon graph in (81) is in one-to-one correspondence with a face of the corresponding 4-simplex, and $\operatorname{sign}\left(f, f^{\prime}\right)$ is defined by the equation (161). The dots in (81) correspond to some symmetric expression that contains only terms with graspings between two edges sharing one vertex.

\subsection{Reisenberger model}

Reisenberger [3] has proposed a state sum model corresponding to the selfdual Plebanski action (43). The main idea of his construction is to modify the SU(2) Crane-Yetter model in such a way that the constraints (44) are implemented. The model can be described as follows. As in the previous subsection, let $\mathcal{M}$ be a triangulated 4-manifold. Let us associate a group element $g_{w}$ to each wedge $w$ of the dual triangulation (see Section 2). One can think of $g_{w}$ as the holonomy of connection around the boundary of the wedge $w$ with the basepoint being the center of the 4-simplex $h$ to which the wedge belongs. Let $D_{w}^{i}$ be a differential su(2) operator acting on functions 
of $g_{w}$ as the sum of left and right invariant vector fields. Let us denote by $\Omega^{i j}$ the following operator acting in $(\mathrm{SU}(2))^{\otimes 10}$ :

$$
\Omega^{i j}=\sum_{w, w^{\prime}} \epsilon\left(w, w^{\prime}\right) D_{w}^{i} D_{w^{\prime}}^{j}-\frac{1}{3} \delta^{i j} \sum_{w, w^{\prime}} \epsilon\left(w, w^{\prime}\right) D_{w}^{k} D_{w^{\prime} k},
$$

where $\epsilon\left(w, w^{\prime}\right)$ is the sign of the four volume span by the wedges $w, w^{\prime}$ $\left(\epsilon\left(w, w^{\prime}\right)=0\right.$ if the two wedges don't span a four-volume). We choose a coloring of the wedges by spins $j_{w}$, denote by $R_{j}(g)$ the representation of $g$ as an endomorphism of $V_{j}$, and define for each 4-simplex $h$ the following function on $(\mathrm{SU}(2))^{\otimes 10}$ :

$$
\phi_{h, \vec{\jmath}}\left(g_{w}\right)=\operatorname{tr}_{\otimes_{w \in h} V_{j_{w}}}\left(\frac{1}{\sqrt{2 \pi x}} e^{-\frac{1}{2 x^{2}} \Omega^{i j} \Omega_{i j}} \otimes_{w \in h V_{j_{w}}}\left(2 j_{w}+1\right) R_{j_{w}}\left(g_{w}\right)\right) .
$$

The state sum model proposed by Reisenberger is given by

$$
\lim _{x \rightarrow 0} \sum_{j_{w}} \int \prod_{w} d g_{w} \prod_{h} \phi_{h, \vec{\jmath}}\left(g_{w}\right)
$$

The limit is a possible way of selecting only the states belonging to the kernel of $\Omega^{i j}$.

\section{Applications of the generating functional}

In this section we use the generating functional computed in Sec.2 to construct systematically state sum models of the theories described in Sec.3. We then compare the results of this systematic approach with the known results described in Sec.4.

\subsection{BF theory: any dimension}

The transition amplitude of the theory is simply the value of the generating function $Z$ at $J=0$. As one can easily see, this gives exactly the PonzanoRegge model (46) in three dimensions, and the Ooguri model (47) in four dimensions. The corresponding two dimensional model is somewhat trivial: the transition amplitude is given by (25) at $J=0$, which gives

$$
\sum_{j}\left(\operatorname{dim}_{j}\right)^{\chi(\mathcal{M})}
$$

where $(\mathcal{M})$ is the Euler characteristics of $\mathcal{M}$. One can recognize in this expression the volume of the space of flat connections on $\mathcal{M}$ modulo gauge transformations expressed in term of Riemann zeta function (see, for example, $[23])$. 


\section{$5.2 \quad 2 \mathrm{D}$ Yang-Mills}

Our general strategy is to calculate the 'interaction' term $\Phi(E)$ of the action (31) on the configuration of $E$ that is distributional along the wedges of the dual complex

$$
E=\sum_{w} E_{w}
$$

express the result as a polynomial function $\Phi(X)$ of the variables $X_{w}$, introduced in the previous section, and then look for the vacuum-vacuum transition amplitude of the theory as given by

$$
\left(e^{i \Phi(-i \delta / \delta J)} Z[J, \Delta]\right)_{J=0} .
$$

In the case of $2 \mathrm{~d}$ Yang-Mills theory, the 'interaction' term $i \Phi$ is given by (see (49))

$$
\frac{e^{2}}{2} \int_{\mathcal{M}} d \mu \operatorname{Tr}\left(E^{2}\right)
$$

Calculating this on the configuration of $E$ given by (86), with each $E_{w}$ being constant along the wedge $w$, we get

$$
\frac{e^{2}}{2} \sum_{w} \rho_{w} \operatorname{Tr}\left(X_{w}^{2}\right)=-e^{2} \sum_{w} \rho_{w} X_{w}^{i} X_{w}^{i}
$$

where $\rho_{w}$ is the area of the wedge $w$, as measured with respect to $d \mu$, and we have introduced the $\mathrm{SO}(3)$ indices (128). The partition function of the theory is then given by

$$
\left(\exp \left(-e^{2} \sum_{w} \rho_{w}\left(\frac{\delta}{\delta i J_{w}^{i}} \frac{\delta}{\delta i J_{w}^{i}}\right)\right) Z[J, \Delta]\right)_{J=0} .
$$

To compare this with the partition function of the Migdal-Witten model, it is more convenient to take the generating functional $Z[J]$ in its general form (22). Then, using

$$
\left.e^{\alpha\left(\frac{\delta}{\delta i J_{w}^{i}} \frac{\delta}{\delta i J_{w}^{i}}\right)} \cdot P(J) R_{(j)}\left(e^{J}\right)\right|_{J=0}=e^{\alpha(j+1 / 2)^{2}}=e^{\alpha(c(j) / 2+1 / 4)}
$$

where $R_{(j)}\left(e^{J}\right)$ is the group element $e^{J}$ taken in the $j$ representation, and $c(j)$ is the quadratic casimir of this representation. We get for the partition function

$$
\prod_{\epsilon} \int d g_{\epsilon} \sum_{j_{\sigma}} \prod_{\sigma} \operatorname{dim}_{j_{\sigma}} \chi_{j_{\sigma}}\left(g_{\epsilon_{1}} \cdots g_{\epsilon_{n}}\right) \exp \left(\sum_{w \in \sigma}-e^{2} \rho_{w}\left(c\left(j_{\sigma}\right) / 2+1 / 4\right)\right) .
$$


This can be seen to be equal to

$$
\mathrm{YM}\left(\rho e^{2}, \mathcal{M}\right) e^{-e^{2} \rho / 4}
$$

by noting that

$$
\sum_{w \in \sigma} \rho_{w}=\rho_{\sigma}, \quad \text { and } \quad \sum_{\sigma} \rho_{\sigma}=\rho .
$$

Thus, our approach gives the correct expression (50) for the partition function of the Yang-Mills theory, apart from the factor $\exp \left(-e^{2} \rho / 4\right)$. However, the later is what is called a "standard renormalization" of the partition function. That is, the result for the partition function of 2D Yang-Mills may depend on a regularization procedure that was used to calculate it, the two different schemes giving results that may differ, in particular, by factors of $\exp \left(-\alpha e^{2} \rho\right)$, where $\alpha$ is some coefficient. See, for instance, [6] for a more detailed discussion of the standard renormalizations. Thus, the partition function obtained by our method differs from that of the Migdal-Witten model just by a standard renormalization factor.

\subsection{D BF theory with cosmological term}

As one can see from (38), the 'interaction' term $i \Phi(E)$ for this theory is given by

$$
i \Phi(E)=-\frac{i \Lambda}{12} \int_{\mathcal{M}} \operatorname{Tr}(E \wedge E \wedge E) .
$$

As in the case of Yang-Mills theory in two dimensions, one has to find a polynomial function $\Phi(X)$ of variables $X_{w}$ by evaluating (94) on the distributional field $E$ given by (2). Unfortunately, in the case of three dimensions the result is not well-defined as in the case of 2D Yang-Mills theory. Indeed, the $E$ field is concentrated along the wedges (see Fig. 7), and non-trivial contributions to the integral (94) come from the points where wedges intersect. Thus, the contributions to (94) come from the integrals

$$
\int_{\mathcal{M}} \operatorname{Tr}\left(E_{w_{1}} \wedge E_{w_{2}} \wedge E_{w_{3}}\right),
$$

where $w_{1}, w_{2}, w_{3}$ are three wedges that intersect. The wedges can intersect at points, as, for example, they do at the center of each tetrahedron, or they can have more general intersections, as, for example, an intersection of three wedges sharing a dual edge. Or, instead, one can have in (95) $w_{1}=w_{2}$. Whatever the type of an intersection is, the result of (95) is ill-defined because of the distributional nature of $E$ field. In this paper we show how the ambiguity in (95) can be resolved for the simplest, and most important type of intersections: intersections of three different wedges at the centers of 
tetrahedra. As we shall see, for intersections of this type there is a simple way to resolve the ambiguity in (95) using geometrical considerations. As we show, this type of intersection is the most important one, for it is responsible for, in certain sense, the most intricate contribution to the transition amplitude of the theory. Thus, in this subsection, we restrict our consideration only to this special type of intersection, calculate the corresponding interaction term $\Phi(X)$, find the corresponding spin foam model, and compare it with the Turaev-Viro model. In the first order in the decomposition of Turaev-Viro amplitude in power of $\Lambda$, we will be able to identify a term analogous to the term we obtain in our model and compare them. We will find a very delicate matching between the two, including the numerical coefficients. The importance of other types of intersections, not treated here, will be emphasized later.

Thus, the intersections we consider are the ones for which three different wedges intersect at the center of a tetrahedron. Given a tetrahedron $t$, there are 20 (without counting the permutations) different triples of wedges $w_{1} \neq w_{2} \neq w_{3}$. Four of these triples do not span a three-volume; thus, it is natural at first to take the integral (95) for such triples to be zero. These are exactly the triples of wedges that share a line - a part of the dual edge. Thus, there are only 16 different triples of wedges (without counting the permutations) that contribute to (95) for a given tetrahedron. Recall now that each $E_{w}$ is given by (27). Thus, for any given triple of wedges $w_{1} \neq w_{2} \neq w_{3}$, the integral (95) is proportional to

$$
i \Lambda \operatorname{Tr}\left(X_{w_{1}} X_{w_{2}} X_{w_{3}}\right),
$$

but the proportionality coefficients are not fixed, because of the indeterminacy in the value of the integral

$$
\int \delta\left(u_{1}\right) \delta\left(u_{2}\right) \delta\left(u_{3}\right) d u_{1} \wedge d u_{2} \wedge d u_{3} .
$$

Of course if the wedges were infinite planes without boundaries this integral is a well-defined quantity: it is just the intersection number and as such should be \pm 1 depending on the orientations of the wedges. Because of the existence of boundary we expect this integral to be a number smaller than 1 . We fix the proportionality coefficient by requiring that

$$
\frac{1}{12} \int_{t} \operatorname{Tr}(E \wedge E \wedge E)
$$

where the integral is taken over a tetrahedron $t$, is equal to the geometrical volume of $t$. For any triple of wedges $w_{1} \neq w_{2} \neq w_{3}$, the integral (97) is equal to $\alpha \cdot \operatorname{sign}\left(w_{1}, w_{2}, w_{3}\right)$, where $\alpha$ is a coefficient that is independent on a 
triple, and $\operatorname{sign}\left(w_{1}, w_{2}, w_{3}\right)$ is plus or minus one depending on the orientation of the form $d u_{1} \wedge d u_{2} \wedge d u_{3}$. Thus, (98) is equal to

$$
\frac{1}{12} \sum_{w_{1} \neq w_{2} \neq w_{3} \in t} \alpha \cdot \operatorname{sign}\left(w_{1}, w_{2}, w_{3}\right) \operatorname{Tr}\left(X_{w_{1}} X_{w_{2}} X_{w_{3}}\right) .
$$

Here the sum is taken over triples of different wedges in $t$, taking into account all different permutations of $w_{1}, w_{2}, w_{3}$. Taking into account the fact that the number of permutations of $w_{1}, w_{2}, w_{3}$ is 6 , we get for (98):

$$
\frac{1}{2} \sum_{w_{1}<w_{2}<w_{3}} \alpha \operatorname{Tr}\left(X_{w_{1}} X_{w_{2}} X_{w_{3}}\right)
$$

where the notation $w_{1}<w_{2}<w_{3}$ means that the sum is taken over 16 different triples $w_{1} \neq w_{2} \neq w_{3}$ such that $\operatorname{sign}\left(w_{1}, w_{2}, w_{3}\right)=1$.

To relate (100) to the volume of tetrahedron $t$, we recall the geometrical interpretation of variables $X_{w}$. They were introduced in the previous section as the variables that carry information about the length of edges of the triangulation. At this stage it is more convenient to introduce $\mathrm{SO}(3)$ indices (128). Thus, each $X_{w}$ is characterized by $X_{w}^{i}, i=1,2,3$. Recall that wedges $w$ are in one-to-one correspondence with edges $e$ of the triangulation. Thus, let us view each $X_{w}^{i}$ as the vector representing the corresponding edge (each edge can be viewed as a vector pointing from one vertex of $\Delta$ to another), and the norm squared $X_{w}^{i} X_{w}^{i}$ of $X_{w}^{i}$ as the length squared of this vector. Indeed, it is not hard to check that the interpretation of $X_{w}^{i} X_{w}^{i}$ as the length of the corresponding edge is consistent with the other known facts. Let us consider the operator corresponding to $X_{w}^{i} X_{w}^{i}$. According to our general prescription, this quantity is represented by the operator $\left(\delta / \delta i J_{w}\right)^{2}$. We have already dealt with this operator in the previous subsection, see (91). Its eigenvalue is given just by the half of the Casimir (plus 1/4). Thus, in the sense of eigenvalues, we can write

$$
X_{w}^{i} X_{w}^{i}=(j+1 / 2)^{2},
$$

where $j$ is the spin from (28) labelling the dual face that contains $w$. Thus, (101) tells us that, in the limit of large spins $j$, the norm of $X_{w}$ grows as $j$. This is to be compared with the length spectrum of the canonical quantum theory

$$
\text { (length) }=\sqrt{j(j+1)}
$$

This also grows as $j$ for large spins. The expression (102) can be easily derived in the context of canonical (loop) quantum gravity in three dimensions (note that we use units in which $8 \pi G=1$ ). Another motivation for 
interpreting the spin $j$ as the length of an edge (for large $j$ ) is that it is exactly this interpretation that must be used to reproduce correctly the Regge calculus version of Einstein-Hilbert action in the Ponzano-Regge model of quantum gravity [7]. Thus, we learn that the interpretation of the norm of each vector $X_{w}$ must be that of the length of the corresponding edge of $\Delta$. Having this fixed we can relate (100) to the volume of tetrahedron $t$. We have

$$
\operatorname{Tr}\left(X_{w_{1}} X_{w_{2}} X_{w_{3}}\right)=2 \epsilon_{i j k} X_{w_{1}}^{i} X_{w_{2}}^{j} X_{w_{3}}^{k},
$$

where we have introduced the $\mathrm{SO}(3)$ indices, see (128). Each $X_{w}^{i}$ has the interpretation of the vector corresponding to one of the edges of $t$. Thus, (103) is equal to $12 V$, where $V$ is the volume of $t$. In (100) we have 16 such terms. Thus, it is equal to

$$
\frac{1}{2} \alpha \cdot 16 \cdot 12 V
$$

The requirement that (98) is equal to the volume of $t$ fixes the parameter $\alpha$ to be $1 /(16 \cdot 6)$. Thus, finally, we obtain the interaction term $i \Phi(X)$ to be

$$
-\frac{i \Lambda}{6} \frac{1}{16} \sum_{w_{1}<w_{2}<w_{3}} \epsilon_{i j k} X_{w_{1}}^{i} X_{w_{2}}^{j} X_{w_{3}}^{k}
$$

where the sum is taken over 16 terms. To obtain the transition amplitude of the theory we have to replace each $X_{w}^{i}$ by the operator $\delta / \delta i J_{w}^{i}$ and act by the exponential of (104) on the generating functional. Thus, the first order term in $\Lambda$ in the decomposition of the transition amplitude is given by:

$$
\left(\left(-\frac{i \Lambda}{24} \frac{1}{16} \sum_{w_{1}<w_{2}<w_{3}} 4 \epsilon_{i j k} \frac{\delta}{\delta i J_{w_{1}}^{i}} \frac{\delta}{\delta i J_{w_{2}}^{j}} \frac{\delta}{\delta i J_{w_{3}}^{k}}\right) Z_{3}(J, \Delta)\right)_{J=0} .
$$

This is to be compared with (67). We will now show that the most complicated term in that expression - the term that involves trivalent graspings - exactly matches our result (105), including the numerical coefficient and the sign. To see this we just have to relate the trivalent grasping in (67) to the cubic operator in (105). As explained in the Appendix D, a single grasping in (67) acts by inserting $\left(\sigma^{i} / \sqrt{2}\right)$, where $\sigma^{i}$ are the Pauli matrices. The operator $\left(\delta / \delta i J^{i}\right)$, when applied only one time, acts by inserting just $\sigma^{i}$. Also, taking into account the definition of the trivalent grasping in (67), one can show that

$$
\mathrm{e}_{\mathrm{e}^{-}-{ }^{\mathrm{e}}}=4 \epsilon_{i j k} \frac{\delta}{\delta i J_{e}^{i}} \frac{\delta}{\delta i J_{e^{\prime}}^{j}} \frac{\delta}{\delta i J_{e^{\prime \prime}}^{k}}
$$


So our approach does account for the most intricate part of the 3D transition amplitude in the first $\Lambda$-order, reproducing correctly even the numerical coefficients. Note, however, that we do not get all of the terms appearing in (67). The discrepancy we find between the model we obtain and the usual state sum model can be understood by comparing the two different expressions for the spacetime volume: the one obtained within our approach and the one obtained within the Turaev-Viro model, see (67). The Turaev-Viro model tells us that we must associate the spacetime volume not only to tetrahedra of the triangulation, but also to edges and vertices. Within our approach, only the tetrahedron part of the spacetime volume is accounted for. The reason for this was that, when evaluating the cosmological term of the action on the distributional $E$ field, we took only the terms which came from intersections of different wedges at the centers of tetrahedra. As we discussed, even these terms are ill-defined because of the distributional nature of the $E$ field. However, the corresponding ambiguities can be successfully resolved for these types of intersections by using geometrical considerations, and, after the ambiguities are eliminated, the result exactly matches the analogous terms of the usual state sum models, including the matching of the numerical factors. The geometrical considerations we used were exactly the ones that relate the terms we considered to the volume of a geometrical 3-simplex. Thus, it is not very surprising that only the "part" of the Turaev-Viro model that accounts for the volume of 3-simplices was reproduced correctly: as the geometrical considerations we used above tell us, we considered only the terms that are relevant for the volume of individual 3simplices. However, there are other types of terms that we did not consider and that may be crucial to reproduce the corresponding state sum model correctly. Let us consider, for example, the terms of the type:

$$
\int_{t} \operatorname{Tr}\left(E_{w_{1}} \wedge E_{w_{1}} \wedge E_{w_{2}}\right)
$$

for some $w_{1} \neq w_{2}$. The result of such an integral is ill-defined. Indeed, there is an indeterminacy of the type $0 \cdot \infty$, where 0 comes from $d u_{1} \wedge d u_{1}$ (see $(27))$, and $\infty$ comes from the square of the $\delta$-function $\delta^{2}\left(u_{1}\right)$. These terms are proportional to

$$
\operatorname{Tr}\left(X_{w_{1}} X_{w_{1}} X_{w_{2}}\right)
$$

Other terms that may arise are

$$
\int_{t} \operatorname{Tr}\left(E_{w} \wedge E_{w} \wedge E_{w}\right)
$$

for some $w$. They arise as the result of the indeterminacy $0^{2} \cdot \infty^{2}$, where $0^{2}$ comes from $d u \wedge d u \wedge d u$ (see (27)), and $\infty^{2}$ comes from the cube of the 
$\delta$-function $\delta^{3}(u)$. These terms are proportional to

$$
\operatorname{Tr}\left(X_{w} X_{w} X_{w}\right) .
$$

There is no obvious reason to set these two types of terms to zero. In fact, as one can see, these are exactly the types of terms that are needed to account for the other terms appearing in the first $\Lambda$-order in the Turaev-Viro model. Although the structure of terms appearing this way is clear, at the present state of the development of our approach, no geometrical arguments is available to fix the ambiguity in the coefficients in front of such terms. However, the precise agreement of the terms for which such arguments do exist gives hope that, ones the above ambiguities are resolved, we will have an exact agreement between the models obtained using our procedure and the usual state sum models.

\subsection{D BF theory with cosmological term}

In the case of $4 \mathrm{D}$ BF theory with cosmological term the interaction $i \Phi$ is given by:

$$
i \Phi(E)=-\frac{i \Lambda}{2} \int_{\mathcal{M}} \operatorname{tr}(E \wedge E) .
$$

Our general prescription is to evaluate this interaction term on the distribution (3) and find a polynomial function $\Phi(X)$. Thus, one has to evaluate integrals

$$
\int_{\mathcal{M}} \operatorname{Tr}\left(E_{w} \wedge E_{w^{\prime}}\right)
$$

with $E_{w}$ given by (29). The integral (108) is non-zero only if wedges $w, w^{\prime}$ intersect. However, similarly to the case of $3 \mathrm{D}$, the result when the wedges intersect is ill-defined because of the distributional nature of $E_{w}$. Thus, again some independent considerations have to be used to fix the ambiguity. We use a strategy similar to the one adopted in the case of 3D BF theory. We consider only the terms coming from wedges $w, w^{\prime}$ intersecting at the center of a 4 -simplex. As we shall see, these are the most important terms in the sense that they are responsible for the main terms in the first order in $\Lambda$ of the Crane-Yetter model. The relevance of other types of terms will be emphasized below. To fix the ambiguity in (108) when $w, w^{\prime}$ are two wedges that intersect at the center of a 4 -simplex $h$ we use geometrical considerations.

Let us consider the integral

$$
-\frac{1}{2} \int_{h} \operatorname{Tr}(E \wedge E)
$$


over the interior of a particular 4-simplex $h$. It is equal to the sum

$$
-\frac{1}{2} \sum_{w, w^{\prime} \in h} \int_{h} \operatorname{Tr}\left(E_{w} \wedge E_{w}^{\prime}\right)
$$

Each of the integrals here is proportional to.

$$
\operatorname{Tr}\left(X_{w} X_{w^{\prime}}\right)=-2 X_{w}^{i} X_{w^{\prime}}^{i}
$$

with the proportionality coefficient given by:

$$
-\frac{1}{2} \int_{h} \delta(u) \delta(v) \delta\left(u^{\prime}\right) \delta\left(v^{\prime}\right) d u \wedge d v \wedge d u^{\prime} \wedge d v^{\prime} .
$$

The later is equal to $-(1 / 2) \alpha \cdot \operatorname{sign}\left(w, w^{\prime}\right)$, where $\alpha$ is a numerical parameter, whose value is not fixed due to the ambiguity referred to above, and $\operatorname{sign}\left(w, w^{\prime}\right)$ is the sign of the volume form in (112). Thus, (109) is equal to

$$
\sum_{w, w^{\prime} \in h} \alpha \cdot \operatorname{sign}\left(w, w^{\prime}\right) X_{w}^{i} X_{w^{\prime}}^{i}
$$

where the sum is taken over wedges $w, w^{\prime}$ inside $h$ that span a 4 -volume. There are exactly 30 terms summed over in (113).

We will fix the parameter $\alpha$ relating the quantities $X_{w}^{i}$ to the geometrical 4-simplex in $\mathbb{R}^{4}$. First, let us note that when $E$ in (109) is equal to the selfdual part of the wedge product of two copies of the frame field:

$$
\begin{aligned}
& E_{a b}={ }^{+} \Sigma_{a b}, \\
& \Sigma_{a b}^{I J}=\theta_{[a}^{I} \theta_{b]}^{J},
\end{aligned}
$$

where $\theta_{a}^{I}$ is a frame (tetrad) field, then (109) is equal to

$$
\begin{aligned}
\int_{h} \frac{1}{2}+\Sigma_{a b}^{i} \frac{1}{2}+\Sigma_{c d}^{i} \tilde{\epsilon}^{a b c d} & =\int_{h} \frac{1}{2}+\Sigma_{a b}^{I J} \frac{1}{2}{ }^{+} \Sigma_{c d I J} \tilde{\epsilon}^{a b c d} \\
& =\frac{1}{16} \int_{h} \epsilon_{I J K L} \Sigma_{a b}^{I J} \Sigma_{c d}^{K L} \tilde{\epsilon}^{a b c d}=\frac{4 !}{16} V_{h},
\end{aligned}
$$

where $V_{h}$ is the volume of $h$ with respect to the metric defined by $\theta_{a}^{I}$. Thus, we will fix $\alpha$ in such a way that (113) is equal to $(3 / 2) V_{h}$ when $X_{w}^{i}$ can be related to the quantities characterizing a geometrical 4 -simplex.

Recall that a geometrical 4-simplex in $\mathbb{R}^{4}$ is characterized (up to translations) by four vectors: the four vectors pointing from one of the vertices to the other four ( for more details see the Appendix E). For each face of $h$ one can also construct the so-called bivectors, which are given by the wedge 
products of any two of the edges belonging to that face. The bivectors live in the second exterior power of $\mathbb{R}^{4}$. One can take the self-dual part of a bivector to obtain an element of $\mathbb{R}^{3}$. As is shown in the Appendix $E$ (see (166)), the norm (obtained using the usual flat metric in $\mathbb{R}^{3}$ ) of the self-dual part of each bivector is equal to the squared area of the corresponding face. There exists also a simple expression (167) for the volume of a 4-simplex involving only the self-dual parts of bivectors. Recall now that wedges $w \in h$ are in one-to-one correspondence with faces of $h$. Then, if $X_{w}^{i}$ has the interpretation of the self-dual part of the bivector corresponding to a face of $h$.

A comparison of (167), (115) fixes the value of $\alpha$ to be $\alpha=1 / 30 \cdot 4$. Thus, the first $\Lambda$-order term of the transition amplitude is given by:

$$
\left(\Lambda \frac{1}{30} \sum_{w, w^{\prime} \in h} \frac{1}{4} \operatorname{sign}\left(w, w^{\prime}\right) \frac{\delta}{\delta i J_{w}^{i}} \frac{\delta}{\delta i J_{w^{\prime}}^{i}} Z(J)\right)_{J=0}
$$

To compare this with (81) we need the relation between the grasping there and the operator in (116). The corresponding relation is given by

$$
\begin{array}{l:l}
\text { e } & =\frac{1}{2} \frac{\delta}{\delta i J_{w}^{i}} \frac{\delta}{\delta i J_{w^{\prime}}^{i}}
\end{array}
$$

Using this correspondence we see that (116) agree with the result (81). As in the $3 \mathrm{D}$ case, the matching includes the numerical coefficient and the sign of the expressions.

Let us now discuss the role of the terms denoted by dots in (81). Let us recall that those terms are determined by a framing of the (15j)-symbol used in the Crane-Yetter model. These terms are given by a sum of graspings of edges of the graph $\Gamma_{h}$ that share a vertex. Recall that vertices of $\Gamma_{h}$ are in one-to-one correspondence with the tetrahedra of $h$. Thus, the general structure of these terms is such that they can be grouped according to a 3simplex (tetrahedron) to which they "belong". Therefore, these terms can be thought of as the contribution to the 4-volume of the BF theory coming from individual tetrahedra of $\Delta$. Thus, these terms are in certain sense analogous to the terms in 3D that carry 3 -volume corresponding to the edges of $\Delta$. As in 3D our approach did not reproduce correctly the terms corresponding to edges, in $4 \mathrm{D}$ we did not reproduce correctly the contribution to the 4volume coming from tetrahedra. Similarly to the case of 3D, these terms may possibly be reproduced if one takes into account the types of intersections in (108) other than the ones considered here. 


\subsection{D Euclidean gravity}

In order to apply our strategy to the self-dual Plebanski model let us consider the "interaction" term of the action (43):

$$
i \Phi(E)=-i \int_{\mathcal{M}} \psi_{i j}\left(E^{i} \wedge E^{j}-\frac{1}{3} \delta^{i j} E^{k} E_{k}\right) .
$$

Using the same procedure as in the case of the 4-dimensional BF theory with cosmological constant, this term, when evaluated on distributional $E$ field, gives

$$
-i \sum_{h} \psi_{i j}\left(v_{h}\right) \Omega_{h}^{i j}(X)
$$

where $v_{h}$ denotes the center of the 4-simplex and

$$
\Omega_{h}^{i j}(X)=\sum_{w, w^{\prime} \in h} \operatorname{sign}\left(w, w^{\prime}\right)\left(X_{w}^{i} X_{w^{\prime}}^{j}-\frac{1}{3} \delta^{i j} X_{w}^{k} X_{w^{\prime} k}\right)
$$

is a quadratic function in the wedge variables $X_{w}$. In order to write (119) we have absorbed some unimportant numerical constants into the Lagrange multipliers $\psi_{i j}$. The generating functional approach tells us that the spin foam transition amplitude of the self dual Plebanski model is given by $\tilde{Z}(0)$ where

$$
\tilde{Z}(J)=\int \prod_{h} d \psi^{i j}\left(v_{h}\right) e^{-i \sum_{h} \psi_{i j}\left(v_{h}\right) \Omega_{h}^{i j}\left(\frac{\delta}{i \delta J}\right)} \cdot Z(J) .
$$

The integration over $\psi^{i j}$ gives rise to delta functions. Thus, $\tilde{Z}(J)$ can be characterized as the solution of

$$
\Omega_{h}^{i j}\left(\frac{\delta}{i \delta J}\right) \tilde{Z}(J)=0
$$

for all 4-simplices $h$, or, equivalently, as the solution of

$$
\left.\Omega_{h}^{i_{1} j_{1}}\left(\frac{\delta}{i \delta J}\right) \cdots \Omega_{h}^{i_{n} j_{n}}\left(\frac{\delta}{i \delta J}\right) \tilde{Z}(J)\right|_{J=0}=0 .
$$

Using the fact that $Z(J)$ and, therefore, $\tilde{Z}(J)$ are gauge invariant and the property that an $s u(2)$ symmetric traceless tensor $\Omega^{i j}$ is totally characterized up to gauge transformation by its square $\Omega^{i j} \Omega_{i j}$ we see that the preceding equation is equivalent to

$$
\left.\left(\Omega_{h}^{i j} \Omega_{i j_{h}}\right)^{n}\left(\frac{\delta}{i \delta J}\right) \cdot \tilde{Z}(J)\right|_{J=0}=0 .
$$

The solution of the later can be written as

$$
\tilde{Z}(0)=\left.\lim _{x \rightarrow 0} \prod_{h} \frac{1}{\sqrt{2 \pi x}} e^{-\frac{1}{2 x^{2}} \Omega_{h}^{i j} \Omega_{i j_{h}}}\left(\frac{\delta}{i \delta J}\right) \cdot Z(J)\right|_{J=0}
$$


In this form the result is very similar to the one given by the Reisenberger model. There are however several differences. The first one is the fact that the derivative operators appearing in our result are the commutative ones, while Reisenberger uses the non-commutative right and left invariant vector fields. The second difference is due to the presence of the factors of $P(J)$ in the generating functional. In the next section we will emphasize significance of these discrepancies.

\section{Discussion}

In this section we summarize what has been achieved, discuss open questions of the approach and give some speculations as to possible directions of future research.

As we have said in the introduction, our main aim was to understand whether the known spin foam (state sum) models, in particular the ones discussed in section 4, could be obtained as a result of some systematic procedure that starts from the corresponding classical action principle. We have proposed such a procedure, motivated by certain results of the loop approach to quantum gravity. Our main idea is two-fold. First, we proposed to include in the path integral certain distributional field configurations. In fact, in this paper we concentrated only on such distributional configurations. The distributional field was taken to be the $E$ field. As we discussed above, this is motivated by the distributional nature of the non-abelian "electric" field of the canonical approach. We put the distributional $E$ field on a collection of two-dimensional polygons in spacetime, for which we used the collection of dual cells (faces) of a fixed triangulation of the spacetime manifold. Second, to calculate the path integral, we have employed the idea of the generating functional. This allows one to discuss different theories from the same point of view. As we have seen, the generating functional is the same for any theory in a given spacetime dimension, and transition amplitudes for different theories arise as different formal power series in the derivatives with respect to the current. Thus, this is quite reminiscent of the standard quantum field theory in Minkowski spacetime picture, where many different theories can arise from the same free theory as sums over different types of Feynman diagrams. As we have seen, the role of the free theory is played in our approach by $\mathrm{BF}$ theory. Below we shall speculate more on the analogy between the diagrammatic expressions arising in our approach and Feynman diagrams.

We have seen that our approach reproduces correctly the transition amplitudes for BF theory in any dimension and the partition function for Yang- 
Mills theory in two dimensions. We have also compared the transition amplitudes for BF theories with cosmological term that arise in our approach with the ones given by the corresponding states sum models. We did this by comparing the first order terms in the decomposition of the corresponding amplitudes in the power series in $\Lambda$. In the case of $4 \mathrm{D}$ theory, we found agreement (apart from the terms that have to do with the framing), and in the 3D case only a partial agreement was reached. Note, however, that our approach does account for the most intricate part of the 3D and 4D transition amplitudes in the first $\Lambda$-order, reproducing correctly even the numerical coefficients. We have also seen, both in the cases of $3 \mathrm{D}$ and $4 \mathrm{D}$, that the missing contributions can be interpreted as coming from singular contributions arising when one regularizes the volume operator. We expect that some new ideas will be necessary to resolve these regularization ambiguities. Presumably, a study of the higher order terms in $\Lambda$ may give some clues as to how these ambiguities must be resolved.

Let us conclude with some speculations and directions for future research. An interesting open question that arises within our approach is as follows. We have seen that the state sum formulation of the topological BF theory can be obtained by using distributional fields associated with a 2-dimensional cellular complex. Moreover, the residual regularized action on the 2-dimensional worldsheet is given by 2D BF-Yang-Mills theory. It is important to understand why such result is valid. One possibility would be to use the degenerate solutions found by Baez [1] as some monopole contributions playing a major role in defining the measure of the field theory. It might be that the topological nature of the theory depends on whether these singular solutions dominate or not the partition function.

In this paper we have discussed only correlation functions in the $E$ fields. It is also possible to use our approach to compute the expectation value of the Wilson loop functionals. We hope to perform such calculations in the future.

Let us now comment on the analogy between the diagrammatic expressions we have found and Feynman diagrams. In our approach the role of the "free" theory is played by the BF theory. Thus, the free transition amplitudes in our case are just the BF transition amplitudes, given by certain sums of products of functions of spins labelling the triangulation. This is to be compared with the Feynman diagram technique free amplitudes which are just lines representing the free propagation of quanta. It is important that the $\mathrm{BF}$ amplitudes are independent of the triangulation used to compute them. They depend only on the properties of the initial and final states (and, of course, on the topology of the manifold that is contained between 
the initial and final hypersurfaces). The more complicated, "interacting" amplitudes are given in our approach by inserting various chord diagrams into spin networks, with spin networks representing the free amplitudes. This is to be compared with how one takes into account interactions in the standard QFT's: graphically this is also represented by inserting certain types of vertices into the free diagrams. In our case, each grasping occurs within a particular simplex of the triangulation. One then has to take a sum over simplices. Similarly, in QFT's (in the coordinate representation) one puts an interaction vertex at a particular point and then integrates over the position of this point. Thus, it is tempting to draw an analogy between a simplex of a triangulation in our case and a point in QFT's. To summarize, spin networks play in our theory the role of the diagrams of free propagation, and the graspings by the chord diagrams are analogous to the insertion of interaction vertices into the diagrams of QFT's.

Finally, let us discuss implications of the results we have obtained for the known spin foam models of quantum gravity in $4 \mathrm{D}$. The most popular of these are the Reisenberger model [3], discussed in some details above, and the Barrett-Crane model [4]. The major problem with both of them is that they are rather invented then derived as a result of some systematic procedure starting from the Einstein-Hilbert action or any equivalent of it. However, as we have seen on the example of the Reisenberger model, and as one can check for the case of Barrett-Crane model, our approach to some extent reproduces the transition amplitudes given by these models. For example, our approach gives a clear interpretation of the so far mysterious evaluation of the spin networks that satisfy the constraint equations on the flat connection that is performed to obtain the amplitude. In our approach this evaluation has an obvious meaning of taking $J=0$ at the end of the calculation of a transition amplitude. We have also seen that the constraint equations of the known spin foam models have the meaning of differential equations that must be satisfied by the generating functional. However, although the main features of the both models were reproduced by our approach, some important details are different in the models we have obtained. Thus, our approach prescribes to use the commuting variational derivatives with respect to the current instead of non-commuting vector fields used by Reisenberger [3]. Also, the presence of the function $P(J)$, which plays a crucial role in, for example, 2D Yang-Mills theory, is not accounted for by the Reisenberger and BarrettCrane models.

One more important lesson that one can learn from our results is as follows. On examples of 3D and 4D BF theories with cosmological term we have seen that the simplest regularization of the "interaction" (volume) term of the action that we have discussed above does not account for all terms 
one gets from the corresponding "exact" models. However, the Reisenberger model, for example, uses exactly the same way of regularizing the constraints of the theory, for, using the terminology of our approach, it takes into account only the simplest types of intersections of wedges when regularizing the constraint part of the action. Our experience with the BF theories tells us that this cannot be correct. Thus, it could be that the problems with the Reisenberger model (for example, no interesting solutions of his constraint equations is known) arise simply because this model does not take into a proper account other terms that arise in the regularization of the constraints. Similar is true for the Barrett-Crane model.

The original motivation for our research program was to find a systematic procedure that gives one a way to derive spin foam models of various theories starting from the corresponding action functionals. However, the procedure proposed in this paper is still far from being able to claim to have achieved this goal. Yet, by studying the procedure proposed, we have learned a lot about existing models and obtained an important insight as to their deep internal structure. Our results also led us to the conclusion that the existing models of quantum gravity in 4D are too oversimplified. In spite of these rather negative conclusions, we believe that some of the insights we have obtained in this paper will become a part of the final, possibly much more elaborate picture.

Acknowledgements: We are grateful to John Barrett, John Baez, Matthias Blau and Jose Zapata for discussions. K. K. was supported in part by the NSF grant PHY95-14240, by Braddock fellowship from Penn State and by the Eberly research funds of Penn State, L.F was supported by the CNRS and a NATO grant.

\section{A Conventions and notations}

We use the following conventions for differential forms and integrals:

$$
\begin{array}{r}
A=\frac{1}{p !} A_{a_{1} \ldots a_{p}} d x^{a_{1}} \wedge \ldots \wedge d x^{a_{p}} \\
\int A_{a_{1} \ldots a_{d}} d x^{a_{1}} \wedge \ldots \wedge d x^{a_{d}}=\int d^{d} x A_{a_{1} \ldots a_{d}} \tilde{\epsilon}^{a_{1} \ldots a_{d}}
\end{array}
$$

where $\tilde{\epsilon}^{a_{1} \ldots a_{d}}$ is the Levi-Civita density, taking the values plus minus one in any coordinate system. Our convention for the curvature form is: $F=$ $d A+A \wedge A$.

Throughout the paper the following symbols will stand for the following 
elements of the triangulation

$$
\begin{gathered}
e-\text { for an edge } \\
f-\text { for a face } \\
t-\text { for a tetrahedron } \\
h-\text { for a } 4 \text {-simplex }
\end{gathered}
$$

We will also use the symbol $\epsilon$ to denote edges of the dual complex (dual 1-cells), and $\sigma$ to denote dual faces (dual 2-cells).

All traces that we use in this paper are in the fundamental representation.

Our $\mathrm{SO}(3)$ index conventions:

$$
\begin{gathered}
X=i \tau^{i} X^{i}, \quad J=\frac{i}{2} \tau^{i} J^{i} . \\
\eta=\frac{\left(q^{1 / 2}-q^{-1 / 2}\right)}{i \sqrt{2 k}} .
\end{gathered}
$$

The quantity $\operatorname{dim}_{q}(j)$ is the so-called quantum dimension of $j \operatorname{dim}_{q}(j)=$ $[2 j+1]_{q}$, where $[n]_{q}$ is the quantum number

$$
[n]_{q}=\frac{q^{n / 2}-q^{-n / 2}}{q^{1 / 2}-q^{-1 / 2}}
$$

having the property that $[k]_{q}=0$.

\section{B Summary of facts on $\mathrm{SU}(2)$}

Here we give a short summary of some standard facts about the group SU(2). One can parameterize an element $g$ of $\mathrm{SU}(2)$ by vectors $Z$ from the Lie algebra. The corresponding relation is given by the exponentiation map:

$$
g=e^{Z}=e^{i \psi n^{i} \sigma^{i} / 2}
$$

where $n^{i}$ is a unit vector $n^{i} n_{i}=1, \psi$ is a real positive parameter, and $\sigma^{i}$ are the usual Pauli matrices:

$$
\left(\sigma^{i} \cdot \sigma^{j}\right)=i \epsilon^{i j k} \sigma^{k}+\delta^{i j}
$$

Thus, $\psi n^{i}$ is an element of $\mathbb{R}^{3} \sim \mathrm{su}(2)$, and (131) gives the exponentiation map. As one can easily check,

$$
g=\cos (\psi / 2)+i n^{i} \sigma^{i} \sin (\psi / 2)
$$


Thus, to cover the whole $\mathrm{SU}(2)$ just ones, the parameter $\psi$ should takes values in the range: $\psi \in[0,4 \pi]$.

The Haar measure on the group can be related to the usual Lebesgue measure in $\mathbb{R}^{3}$ by introducing a function $P(Z)$ on the Lie algebra:

$$
P(Z)=\left(\frac{\sin (\psi / 2)}{\psi / 2}\right) .
$$

Then $P^{2}(Z) d Z / 32 \pi^{2}$ gives the normalized Haar measure on $\mathrm{SU}(2)$ in terms of the Lebesgue measure $d Z$.

The characters of irreducible representations are given by:

$$
\chi_{j}\left(e^{Z}\right)=\frac{\sin (\psi(j+1 / 2))}{\sin (\psi / 2)},
$$

where $j$ are half-integers (spins).

The Fourier transform on SU(2) maps any function on the group into a function on the space dual to the Lie algebra. Let $f$ be a coordinate on the space $\operatorname{su}(2)^{*}$. Then the Fourier transform is given by:

$$
\tilde{\phi}(f):=\int \frac{d Z}{V} P(Z) e^{-i f(Z)} \phi(\exp Z) .
$$

The inverse Fourier transform is given by:

$$
\phi(\exp Z)=\sum_{j} \operatorname{dim}_{j} \frac{1}{P(Z)} \int_{j} d \Omega_{f} e^{i f(Z)} \tilde{\phi}(f),
$$

where the integrals are taken over the co-adjoint orbits - spheres of radius $j+1 / 2$, and the measure $d \Omega_{f}$ on each orbit is normalized so that

$$
\int_{j} d \Omega_{f}=\operatorname{dim}_{j}=2 j+1 \text {. }
$$

A particular case of (137) is the following simple formula for characters (135):

$$
\chi_{j}\left(e^{Z}\right)=\frac{1}{P(Z)} \int_{j} d \Omega_{f} e^{i f(Z)} .
$$

This is the famous Kirillov formula [27].

\section{Haar measure, intertwiners and spin networks}

Let us denote by $V_{i}$ the spin $i$ representation of SU(2) and by $V_{i}^{*}$ the dual representation, which in the case of $\mathrm{SU}(2)$ is isomorphic to $V_{i}$. Let us denote 
by $\epsilon_{i}$ the corresponding isomorphism, and by $R_{i}(g)$ the representation of the group element $g$ in $V_{i}$.

Throughout the paper we use the normalized Haar measure

$$
\int d g=1
$$

Let $K$ be an intertwiner between the trivial representation and a representation $V$, that is $K \in H_{o m}(\mathbb{R}, V)$. We will denote by $\bar{K}$ its dual $\bar{K} \in \operatorname{Hom}_{G}\left(V, \mathbb{R}^{*}\right)$. Intertwiners are the basic building blocks of the socalled spin networks. Another usage of the intertwiners is to express the result of integration of a product of group elements. Let us denote by $K_{\alpha}^{i_{1}, \ldots, i_{n}}$ an orthonormal basis of $H_{o m}\left(\mathbb{R}, V_{i_{1}} \otimes \cdots \otimes V_{i_{n}}\right)$. The intertwiners $K_{\alpha}^{i_{1}, \ldots, i_{n}}$ have the property that

$$
\bar{K}_{\alpha} K_{\beta}=\delta_{\alpha, \beta},
$$

where the product between $\bar{K}$ and $K$ is defined by the duality bracket $V \otimes$ $V^{*} \rightarrow \mathbb{R}$. Then the integral of $n$ group elements is given by

$$
\int d g R_{i_{1}}(g) \otimes \cdots \otimes R_{i_{n}}(g)=\sum_{\alpha} K_{\alpha}^{i_{1}, \ldots, i_{n}} \bar{K}_{i_{1}, \ldots, i_{n}}^{\alpha},
$$

where $R_{i}(g)$ is considered as an element of $V_{i} \otimes V_{i}^{*}$. To integrate a product where both $g$ and $g^{-1}$ appear one has to use the duality relation $\bar{R}_{i}(g)=$ $R_{i}\left(g^{-1}\right)=\epsilon_{i} R_{i}(g) \epsilon_{i}^{*}$. For instance

$$
\int d g\left(\bar{R}_{i}\right)_{m n}\left(R_{j}\right)_{m^{\prime} n^{\prime}}=\frac{1}{\operatorname{dim}_{j}} \delta_{i j} \delta_{m m^{\prime}} \delta_{n n^{\prime}}
$$

This equality can be conveniently expressed graphically if one represents a matrix element $\left(R_{i}\right)_{m n}$ by a line labelled by $i$ with the two open ends corresponding each to one of the indices $m, n$. Let us symbolically denote the integration over the group by a circle going around the lines representing the group elements. Then (142) takes the following form:

$$
\prod^{j}=\frac{1}{\operatorname{dim}_{i}} \delta_{i j}{ }^{i}
$$

To find a graphical representation of the result of the integrals involving more than two matrix elements we introduce a trivalent vertex - analog of the Clebsch-Gordan symbol - normalized in a special way. But first, let us define the so-called spin networks. 
An SU(2) spin network functional is defined by the following data: (i) an oriented graph $\Gamma$; (ii) a map $\mathbf{j}$ from the set of edges of $\Gamma$ to the set of irreducible representations of $\mathrm{SU}(2)$; (iii) a map i from the set of vertices $V$ to the set of intertwiners, which assigns to each vertex $v$ an intertwiner from the tensor product of representations labelling the incoming edges to the tensor product of representations labelling the outgoing edges. A spin network is a function on a number of copies of $\mathrm{SU}(2)$, more precisely, on $(\mathrm{SU}(2))^{E}$, where $E$ is the number of edges in $\Gamma$. To find the value of this function one takes for each edge the matrix element of the group element on that edge in the representation that labels the edge, and contracts the matrix elements corresponding to all edges using intertwiners at vertices. The function obtained this way is gauge invariant.

In this paper we will use the normalized trivalent vertex, defined in such a way that the $\theta$-symbol constructed from 3 spins is always equal to unity:

$$
(\mathrm{i}, \mathrm{j} / \mathrm{k}))_{0}=1,
$$

where the operation $(\cdot)_{0}$ denotes the evaluation of the corresponding spin network on all group elements equal to the unity in the group.

With this normalization of the trivalent vertex intertwiner, one can show that the following relation holds:

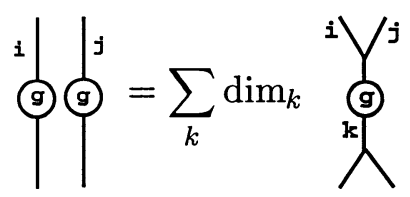

Using this relation one can find the result of the integral of a product of 3 and 4 group elements. The corresponding formulas are:

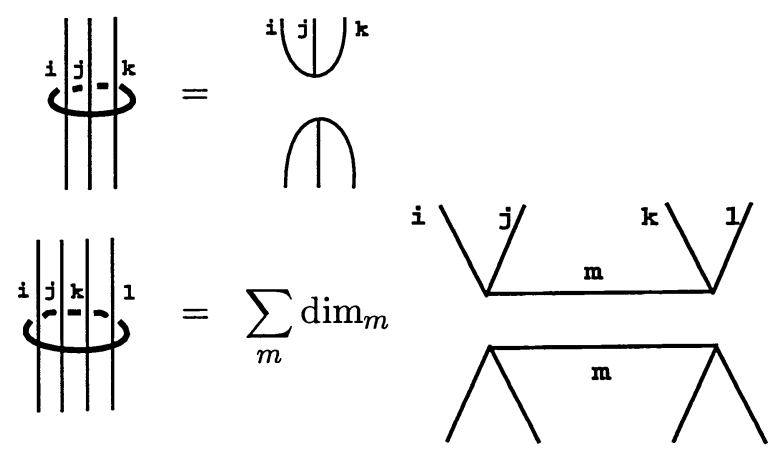

Also, we use the same trivalent vertex to define the normalized (6j),(15j)symbols used in the body of the paper. Both symbols are given by evalu- 
ations of the corresponding spin networks, where the intertwiner used in a trivalent vertex is always the one normalized as in (144). Thus, we get:

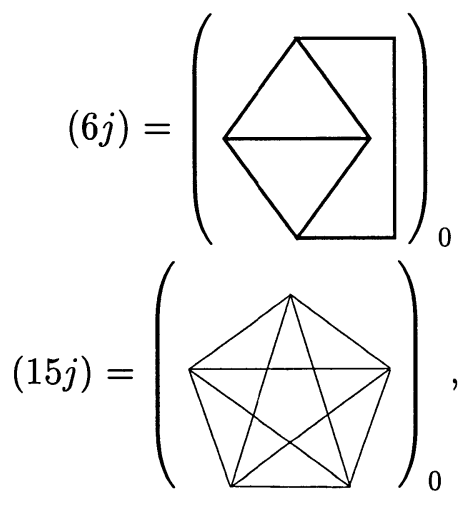

where the resolution of the 4-valent vertex in (149) is given by:

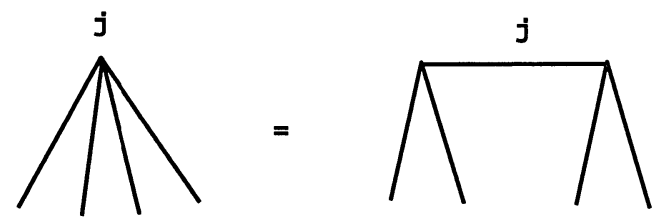

\section{Quantum 6j symbol}

Let $X$ be a one dimensional oriented compact manifold, or more generally an oriented graph. A chord diagram (usually referred as Chinese character chord diagram) with support $X$ is the union $D=\bar{D} \cup X$ where $\bar{D}$ is a graph with univalent and trivalent vertices, together with a cyclic orientation of trivalent vertices and such that univalent vertices lie in $X$. Trivalent vertices are referred to as internal vertices, and the degree of $D$ denoted by $d^{\circ}(D)$ is half the number of vertices of the graph $\bar{D}$. Let $\tilde{\mathcal{A}}_{n}$ be the $\mathbf{Z}$ module freely generated by chord diagrams of degree $n$. We define the $\mathbf{Z}$ module of Vassiliev diagrams of degree $\mathrm{n}$ denoted by $\mathcal{A}_{n}$ as being the quotient of $\tilde{\mathcal{A}}_{n}$ by the relations (STU, IHX, AS) shown in Figure 8. In the figures we always represent the support $X$ with bold lines and the graph $\bar{D}$ with dashed chords.

Given a Lie group $G$, and a coloring $C$ of the graph it is possible to define an evaluation of chord diagrams that is usually called weight system and denoted by $\omega_{G, C}$. Here $C$, the coloring of the graph $X$, is a map which assigns a representation of $G$ to any edge of the graph and an intertwiner to any vertex of the graph $X$. Given a chord diagram $D$ with support $X$ and a coloring $C$ of $X$, we define $\omega_{G, C}(D)$ as shown in Figure 9. Here $X_{a}$ 

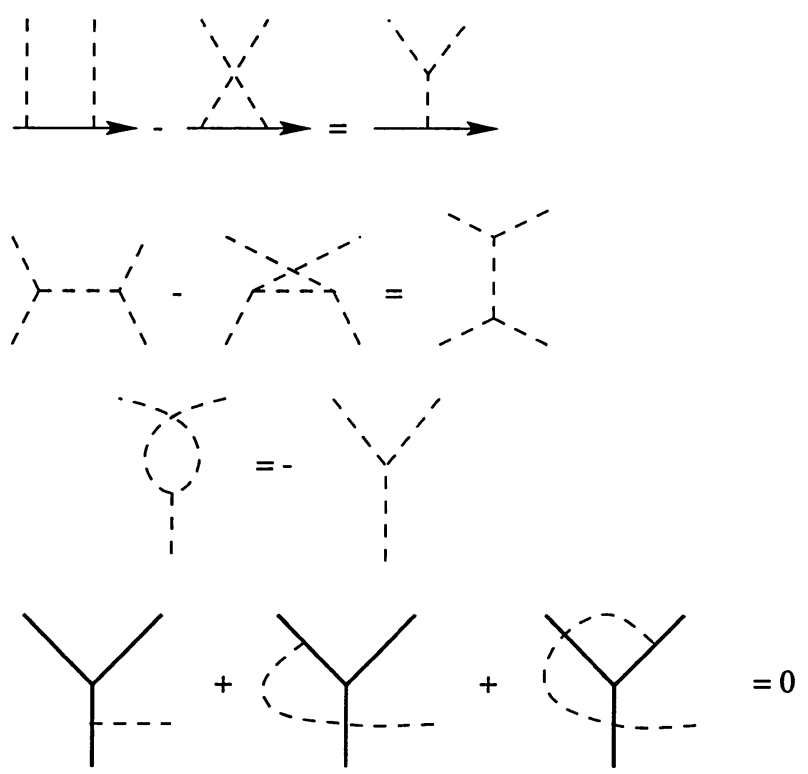

Figure 8:

denotes a basis of the Lie algebra of $G, f^{a b c}$ denotes the structure constants associated with each internal vertices and $t=t^{a b} X_{a} \otimes X_{b}$ is the quadratic casimir $t=t^{a b} X_{a} \otimes X_{b}$ associated to each chord.

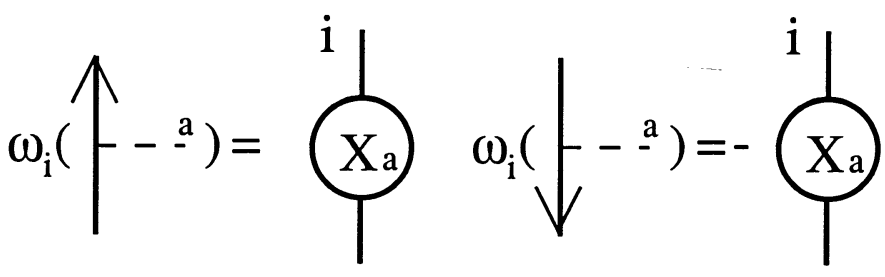

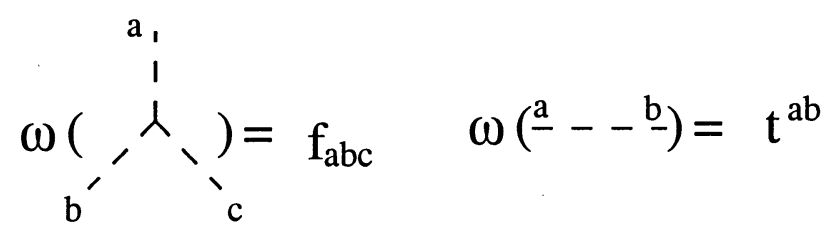

Figure 9:

The space of chord diagrams has been used to define the now famous universal invariant of framed link $Z$ [28], which assigns to any link $L$ in $R^{3}$ a formal power series in $\hbar Z(L)=\sum_{n} \hbar^{n} Z_{n}(L)$, with $Z_{n}(L) \in \mathcal{A}_{n}$. We are not going into the details of this construction. However we must say that this construction uses three main building blocks: The braiding $R \in \mathcal{A}\left(I^{2}\right)$, the associator $\phi \in \mathcal{A}\left(I^{3}\right)$, which should satisfy the pentagon and hexagon 
identity, and the value of the un-knot $\nu \in \mathcal{A}(I)$, where $I$ denotes the unit interval $(151)[29,30]$.

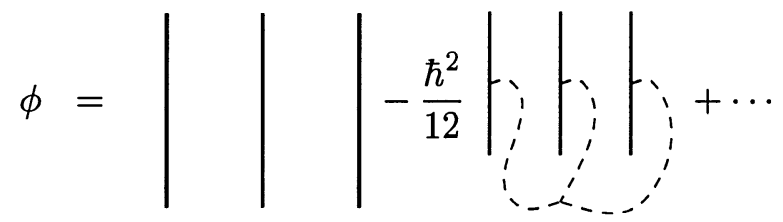

$$
\begin{aligned}
& R=|\quad|+\frac{i \hbar}{2}|---|-\frac{\hbar^{2}}{2}|----|+\cdots \\
& \nu=\mid-\frac{\hbar^{2}}{12} \underset{\substack{--1 \\
-1}}{-i}
\end{aligned}
$$

One important property of the universal invariant is that $\omega_{G, C}(Z(L))$ is equal to the Reshetikhin-Turaev evaluation of the link $L$ associated with the quantum group $U_{q}(G)[31,30,32]$, where $q=\exp i \hbar$ and the casimir is normalized such that the norm of long roots is 2 .

It has been shown recently [33,34], that the universal invariant $Z$ can be extended to an invariant of oriented, framed trivalent graphs embedded into $R^{3}$. The evaluation of the tetrahedral graph with the universal invariant is given by equation (154).

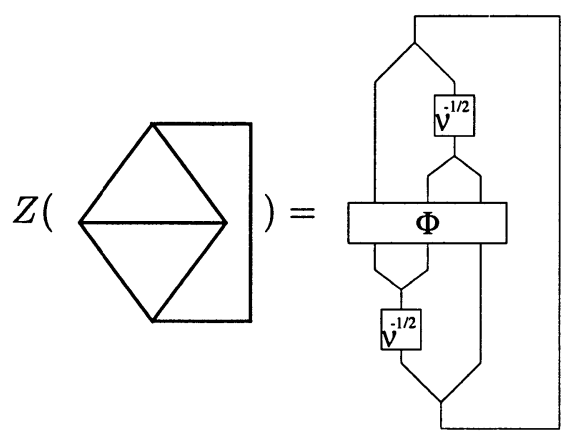

If we evaluate the result of Fig. 154 using the weight system $\omega_{s u(2), C}$ we get the normalized $6 j$ symbol expanded in terms of $\hbar$ (Reshetikhin-Turaev evaluation of the tetrahedral graph for $\left.U_{e^{i \hbar}}(s u(2))\right)$. The term proportional to $\hbar^{0}$ is just the classical $6 j$ symbol. The next term, proportional to $\hbar^{2}$, is 


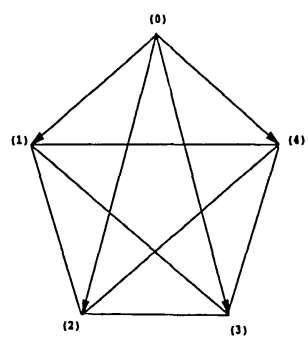

Figure 10: A 4-simplex is characterized up to translations by four vectors.

given by :

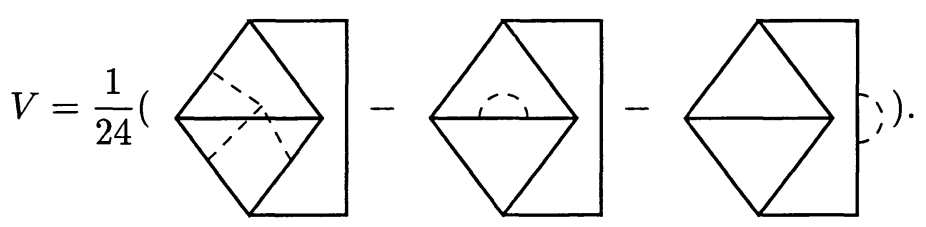

Here we have used the fact that, in the case of $s u(2)$ and for the normalization of the casimir given by the trace in the fundamental representation, the identity $\phi=4$ is satisfied. This term can be written in a symmetric form as:

$$
t e t^{(1)}=\frac{1}{16}\left(\frac{1}{24} \sum_{e, e^{\prime}, e^{\prime \prime}} \begin{array}{l}
\mathrm{e}_{\mathrm{e}}, \ldots \\
\mathrm{e}^{\prime}, \cdots
\end{array}\right.
$$

where the first sum is over all triples of distinct edges of the tetrahedra and the second sum is over all edges of the tetrahedra.

\section{E Geometric 4-simplex}

In this Appendix we list some facts about the geometry of a 4-simplex in $\mathbb{R}^{4}$. The geometrical considerations used in (5.4) are based on some of these facts.

A 4-simplex in $\mathbb{R}^{4}$ is characterized (up to translations) by four vectors. These, for instance, can be vectors pointing from one of the vertices, which we will denote by (0), to the other four vertices (1)-(4). See Fig. 10. Let us denote these vectors by $e_{a}^{I}$. Here $I=1 \ldots 4$ is an index for a vector in $\mathbb{R}^{4}$, and $a=1 \ldots 4$ indicates a vertex at which the vector is directed. Thus, $e_{1}^{I}$ is a vector pointing from vertex (0) to the vertex (1). 
Instead of vectors it is sometimes more convenient to use the so-called bivectors. A bivector $E^{I J}$ is an element of the second exterior power of $\mathbb{R}^{4}$. In particular, it can be obtained by wedging two vectors. Thus, bivectors that characterize a 4 -simplex are obtained as $E_{a b}^{I J}=e_{a}^{[I} e_{b}^{J]}$. Here the brackets denote the operation of antisymmetrization. It is not hard to see that bivectors $E_{a b}^{I J}$ are in one-to-one correspondence with faces of the 4-simplex $h$. For example, the bivector $E_{12}^{I J}$ corresponds to the face whose vertices are $(0),(1),(2)$. The norm of each bivector is proportional to the squared area of the corresponding face

$$
E_{a b}^{I J} E_{a b I J}=2 A_{a b}^{2},
$$

where $A_{a b}$ is the area of the face (0),(a),(b) and no summation over $a, b$ is assumed. The volume of $h$ can be obtained by wedging two bivectors that correspond to faces that do not share an edge. For example, with our choice of orientation of $\mathcal{M}$, the volume is given by

$$
V_{h}=\frac{1}{4 !} \epsilon_{I J K L} E_{12}^{I J} E_{34}^{K L} .
$$

It is sometimes convenient to introduce bivectors corresponding to all 10 faces of $h$. So far we have introduced 6 bivectors $E_{a b}^{I J}$ corresponding to 6 faces of $h$. Bivectors that correspond to other 4 faces can be obtained as linear combinations of these 6 bivectors. When working with all 10 bivectors, it is convenient to label bivectors by 3 different indices instead of just two. We will employ for this purpose small Greek letters. Thus, bivectors are denoted by $E_{\alpha \beta \gamma}^{I J}, \alpha, \beta, \gamma=0 \ldots 4$. These bivectors are defined by

$$
E_{\alpha \beta \gamma}^{I J}=e_{\alpha \beta}^{[I} e_{\alpha \gamma}^{J]}
$$

where $e_{\alpha \beta}^{I}$ is a vector that points from the vertex $\alpha$ to vertex $\beta$. The norm of all bivectors (159) is equal to the twice of the squared area of the corresponding face, as in (157). As we have said above, only 6 of 10 bivectors (159) are independent. Thus, there are certain relations between them. One can write one such relation for each tetrahedron of $h$. One gets 5 relations only 4 of which are independent. With our definition (159) these relations are

$$
\begin{aligned}
& E_{012}+E_{023}-E_{013}-E_{123}=0 \\
& E_{013}+E_{034}-E_{014}-E_{134}=0 \\
& E_{024}+E_{234}-E_{023}-E_{034}=0 \\
& E_{014}+E_{124}-E_{012}-E_{024}=0 \\
& E_{123}+E_{134}-E_{124}-E_{234}=0
\end{aligned}
$$


where we have suppressed the indices $I, J$ for brevity. The volume of $h$ can be expressed as a wedge product of any two of the bivectors (159) corresponding to faces that do not share an edge. This can be written as

$$
\operatorname{sign}\left(f, f^{\prime}\right) V_{h}=\frac{1}{4 !} \epsilon_{I J K L} E(f)^{I J} E\left(f^{\prime}\right)^{K L},
$$

where we have introduced a notation $E(f)^{I J}$ for a bivector that corresponds to face $f$, and $\operatorname{sign}\left(f, f^{\prime}\right)$ is the sign of the right-hand-side in (161). The expression (161) gives the volume of $h$ for any two pairs of faces $f, f^{\prime}$ that do not share an edge. One can use the expression (158) and the "closure" relations (160) to work out the correct sign of any of such formula for the volume.

Any bivector can naturally be split in its self-dual and anti-self-dual parts:

$$
E_{\alpha \beta \gamma}={ }^{+} E_{\alpha \beta \gamma}+{ }^{-} E_{\alpha \beta \gamma} .
$$

The self-dual and anti-self-dual parts are given correspondingly by

$$
\begin{aligned}
{ }^{+} E_{\alpha \beta \gamma} & =\frac{1}{2}\left(E_{\alpha \beta \gamma}+{ }^{*} E_{\alpha \beta \gamma}\right), \\
{ }^{-} E_{\alpha \beta \gamma} & =\frac{1}{2}\left(E_{\alpha \beta \gamma}-{ }^{*} E_{\alpha \beta \gamma}\right),
\end{aligned}
$$

where the Hodge star duality operation is defined as

$$
{ }^{*} E_{\alpha \beta \gamma}^{I J}=\frac{1}{2} \epsilon \epsilon_{K L}^{I J} E_{\alpha \beta \gamma}^{K L} .
$$

Since the space of self-dual (and anti-self-dual) bivectors in $\mathbb{R}^{4}$ is threedimensional, we can introduce a new set of indices to label them. Thus, as the index for self-dual (anti-self-dual) bivector we will use lower case Latin letters from the middle of the alphabet: $i, j, k, \ldots=1,2,3$. The norm of any self-dual (anti-self-dual) bivector calculated by contracting indices $I, J$ will be the same as the norm calculated by contracting the single index $i$ :

$$
{ }^{+} E^{i+} E_{i}={ }^{+} E^{I J+} E_{I J},
$$

where we have suppressed the indices $\alpha, \beta, \ldots$

Not any bivector in $\mathbb{R}^{4}$ is simple, that is, not any bivector is a wedge product of two vectors. The necessary and sufficient requirement of simplicity is that the norm of the self-dual part is equal to the norm of the anti-self-dual part of the bivector. Thus, using (157), we can conclude that when a bivector is simple, its self-dual part norm is equal to the squared area of the corresponding face.

$$
{ }^{+} E_{\alpha \beta \gamma}^{i}{ }^{+} E_{\alpha \beta \gamma i}=A_{\alpha \beta \gamma}^{2} .
$$


There exists an expression for the volume of $h$ that involves only the self-dual parts of bivectors (159):

$$
V_{h}=\frac{1}{3 !} \frac{1}{30} \sum_{f, f^{\prime}} \operatorname{sign}\left(f, f^{\prime}\right)^{+} E^{i}(f)^{+} E_{i}\left(f^{\prime}\right)
$$

where ${ }^{+} E^{i}(f)$ is the self-dual part of the bivector (159) corresponding to the face $f$, the sum is taken over all pairs $f, f^{\prime}$ of faces that do not share an edge, and $\operatorname{sign}\left(f, f^{\prime}\right)$ is the function introduced above by equation (161). The factor of $1 / 30$ in (167) appears to cancel the factor that comes from the sum over 30 terms $f, f^{\prime}$.

\section{References}

[1] J. Baez, Spin foam models, Class. Quant. Grav. 15 (1998), 1827-1858.

[2] M. Reisenberger and C. Rovelli, "Sum over surfaces" formulation of loop quantum gravity, Phys. Rev. D56 (1997), 3490-3508.

[3] M. Reisenberger, On a simplicial model of 4-d general relativity, talk given at Penn State, April 1996;

A lattice worldsheet sum for 4-d Euclidean general relativity, preprint available as gr-qc/9711052.

[4] J. Barrett and L. Crane, Relativistic spin networks and quantum gravity, J. Math. Phys. 39 (1998), 3296-3302.

[5] A. Migdal, Recursion equations in gauge theories, Sov. Phys. JETP 42 (1975), 413

[6] E. Witten, On quantum gauge theories in two dimensions, Commun. Math. Phys. 141 (1981), 153-209.

[7] G. Ponzano and T. Regge, Semiclassical limits of Racach coefficients, In Spectroscopic and theoretical methods in physics, ed. F. Block, NorthHolland Amsterdam, 1968.

[8] V. G. Turaev, O. Y. Viro, State sum invariants of 3-manifolds and quantum 6j-symbols, Topology 31 (1992), N4, 865-902.

[9] H. Ooguri, Topological lattice models in four dimensions, Mod. Phys. Lett. A7 (1992), 2799-2810.

[10] L. Crane, L. Kauffman, D. Yetter, State-sum invariants of 4-manifolds, preprint available as hep-th/9409167. 
[11] L. Freidel, K. Krasnov and R. Puzio, BF description of higherdimensional gravity theories, hep-th/9901069.

[12] C. Rovelli and L. Smolin, Loop representation for quantum general relativity, Nucl. Phys. B331 (1990), 80-152.

[13] C. Rovelli and L. Smolin, Spin networks in quantum gravity, Phys. Rev. D52 (1995), 5743-5759.

[14] J. Baez, Generalized measures in gauge theory, Lett. Math. Phys. 31 (1994), 213-223;

A. Ashtekar and J. Lewandowski, Projective techniques and functional integration, J. Math. Phys. 36 (1995), 2170-2191.

[15] A. Ashtekar and J. Lewandowski, Quantum theory of geometry I: area operators, Class. Quant. Grav. 14 (1997), A55-A81;

Quantum theory of geometry II: volume operators, Adv. Theor. Math. Phys. 1 (1998), 388-429.

[16] T. Regge, General relativity without coordinates, Nuov. Cim. 19 (1961), 558-571.

[17] J. Baez, Degenerate solutions of general relativity from topological field theory, Commun. Math. Phys. 193 (1998), 219-231.

[18] J. Barrett, The path integral for three-dimensional gravity, manuscript in preparation.

[19] P. Dirac, The theory of magnetic poles, Phys. Rev. 74 (1948), N7, 817-830;

The monopole concept, Int. Journ. Theor. Phys. 17 (1978), N4, 235247.

[20] A. M. Polyakov, Quark confinement and topology of gauge theories, Nucl. Phys. B120 (1977), 429-458.

[21] F. Fucito, M. Martellini, M. Zeni, The BF Formalism for QCD and Quark Confinement, Nucl. Phys. B496 (1997), 259-284.

A. S. Cattaneo, P. Cotta-Ramusino, F. Fucito, M. Martellini, M. Rinaldi, A. Tanzini, M. Zeni, Four-Dimensional Yang-Mills Theory as a Deformation of Topological BF Theory, Comm. Math. Phys. 197 (1998), 571-621

[22] M. Reisenberger, A left-handed action for Euclidean general relativity, Class. Quant. Grav. 14 (1997), 1753-1770. 
[23] M. Blau and G. Thompson, Lectures on 2d Gauge Theories, Topological aspects and Path Integral Techniques, available as hep-th/9310144.

[24] J. F. Plebanski, On the separation of Einsteinian substructures, J. Math. Phys. 18 (1977), 2511.

T. Jacobson and L. Smolin, Covariant action for Ashtekar's form of canonical gravity, Class. Quant. Grav. 5 (1988), 583.

R. Capovilla, J. Dell, T. Jacobson, L. Mason, Self-dual two forms and gravity, Class. Quant. Grav. 8 (1991), 41-57.

[25] J. Roberts, Skein Theory and Turaev-Viro Invariants, Topology 34, 771-787 (1995).

[26] L. Freidel and K. Krasnov, Discrete space-time volume for 3-dimensional BF theory and gravity, Class. Quant. Grav. 16 (1999), 351-362.

[27] A. A. Kirillov, Elements of the theory of representations, New-York, Springer-Verlag (1976).

[28] M. Kontsevich, Vassiliev's knot invariants, Adv. in Sov. Math. 16(2) (1993), 137-150.

[29] V. G. Drinfeld, Quasihopf algebras and the Kniznik-Zamolodchikov equations, in Problems of modern quantum field theory, ed. A. Belavin et al., Springer (1990).

[30] P. Cartier, Construction combinatoire des invariants de VassilievKontsevich des nœuds, C. R. Acad. Sci. Paris 316 Série I (1993), 12051210 .

[31] D. Altschuler, L. Freidel, On universal Vassiliev invariants, Comm. Math. Phys. 170 (1995), 41-62.

[32] T. Q. Le, H. Murakami, J. Murakami, The universal VassilievKontsevich invariant for framed oriented links, Compositio Math. 102 (1996), 42-64.

[33] J. Murakami, T. Ohtsuki, Topological quantum field theory for the universal quantum invariant, Comm. Math. Phys. 188 (1997), 501520 .

[34] L. Freidel, in preparation. 